\title{
TU/e EmonOWEN

\section{A novel plasma position and shape controller for advanced configuration development on the TCV tokamak}

\section{Citation for published version (APA):}

Anand, H., Coda, S., Felici, F., Galperti, C., \& Moret, J. M. (2017). A novel plasma position and shape controller for advanced configuration development on the TCV tokamak. Nuclear Fusion, 57(12), [126026].

https://doi.org/10.1088/1741-4326/aa7f4d

DOI:

10.1088/1741-4326/aa7f4d

Document status and date:

Published: 22/09/2017

\section{Document Version:}

Accepted manuscript including changes made at the peer-review stage

\section{Please check the document version of this publication:}

- A submitted manuscript is the version of the article upon submission and before peer-review. There can be important differences between the submitted version and the official published version of record. People interested in the research are advised to contact the author for the final version of the publication, or visit the $\mathrm{DOI}$ to the publisher's website.

- The final author version and the galley proof are versions of the publication after peer review.

- The final published version features the final layout of the paper including the volume, issue and page numbers.

Link to publication

\section{General rights}

Copyright and moral rights for the publications made accessible in the public portal are retained by the authors and/or other copyright owners and it is a condition of accessing publications that users recognise and abide by the legal requirements associated with these rights.

- Users may download and print one copy of any publication from the public portal for the purpose of private study or research.

- You may not further distribute the material or use it for any profit-making activity or commercial gain

- You may freely distribute the URL identifying the publication in the public portal.

If the publication is distributed under the terms of Article 25fa of the Dutch Copyright Act, indicated by the "Taverne" license above, please follow below link for the End User Agreement:

www.tue.nl/taverne

Take down policy

If you believe that this document breaches copyright please contact us at:

openaccess@tue.nl

providing details and we will investigate your claim. 


\title{
A novel plasma position and shape controller for advanced configuration development on the TCV tokamak
}

\author{
H. Anand ${ }^{1}$, S. Coda ${ }^{1}$, F. Felici ${ }^{2}$, C. Galperti ${ }^{1}$, and J.- M. Moret ${ }^{1}$ \\ ${ }^{1}$ École Polytechnique Fédérale de Lausanne (EPFL), Swiss Plasma \\ Center (SPC), EPFL SB SPC, Station 13, CH-1015 Lausanne, \\ Switzerland. \\ ${ }^{2}$ Eindhoven University of Technology, Mechanical Engineering, \\ Control Systems Technology. P.O. Box 513, 5600 MB Eindhoven, \\ The Netherlands.
}

July 3, 2017

\begin{abstract}
A novel plasma position and shape controller has been developed for the highly flexible shaping poloidal-field coil set of the TCV tokamak, to aid in the precise control of advanced configurations such as negativetriangularity plasmas, snowflake and super-X divertors, and doublets. This work follows and relies on the deployment of a new, sub-ms, real-time magnetic equilibrium-reconstruction algorithm. The controller formulation ensures flexibility through an ordering of controlled variables from the most easily to the least easily controlled, while respecting the hardware limits on the poloidal-field coil currents. A rigid, linearised plasma response model for the TCV tokamak is used for the verification and determination of the control parameters. The controller has been applied successfully to a variety of TCV plasma discharges.
\end{abstract}

\section{Introduction}

In modern tokamak machines the plasma position and shape control system is one of the crucial tool to achieve the desired performance. Indeed, being vertically unstable the elongated advanced configurations currently run on tokamaks requires active plasma position stabilization. Additionally, in order to obtain the best performance from a given device, it is necessary to maximise the plasma 
volume within the available space; hence, the ability to control the shape of the plasma while ensuring adequate clearance between the plasma and the plasmafacing wall components is a crucial asset in modern tokamak operation [1]. In tokamaks, the shape of the plasma cross-section is observed to strongly influence a wide range of plasma properties, such as the plasma pressure and current limits [2] and the sawtooth stability [3, 4]. Experimental investigations in TCV have revealed that the energy confinement time (the ratio of the energy stored in the plasma to the power used to heat it, a confinement figure of merit) nearly doubles when going from positive to negative triangularity [5]. Accurate shape control can not only take full advantage of such properties, but can also allow detailed comparisons between experiment and modeling and testing of theoretical models of plasma stability and confinement. Plasma shaping is also effective in stabilising MHD modes and preventing disruptions, especially in the current ramp-up, which is of key importance for the ITER high-currents scenarios. Active plasma shaping thus has a direct impact on MHD stability and on heat and particle transport.

On the TCV tokamak, plasma shaping is especially challenging, as the device features a highly elongated vessel that does not intrinsically constrain the plasma shape and is specifically designed for shape versatility, with a highly flexible poloidal-field coil system. Indeed, TCV accommodates a large variety of plasma shapes, together with various divertor configurations, including single and double null divertor with a broad range of strike point positions and flux expansions. This flexibility has been further extended in recent years to high order null points with more than two strike points such as in the so called 'snowflake' divertor [6]. Even more recently, other advanced configurations such as the super-X, $\mathrm{X}$-divertor, and X-point-target divertor have been explored in TCV [7, 8].

Until now, the TCV plasma shape has been controlled almost entirely in feedforward mode, with the exception of a linearised elongation controller employing a simplified estimator, used in some discharges [9] and some experiments with robust MIMO controller for controlling the plasma shape [10]. A real-time control algorithm capable of not only providing control of plasma position but also high order shape moments, X-points and strike points would thus be highly desirable for optimizing performance in future TCV campaigns. Deviations from the assumptions used in calculating the feedforward parameters can and do cause departures from the desired shape in the absence of feedback control. This happens in particular as a result of auxiliary heating and current drive altering the current profile. Feedback control of plasma shape is also clearly superior to pure feedforward control in its ability to deal with disturbances [11, 12, 13, 14, 15]. Success in controlling plasma shapes in feedforward mode in TCV has often been achieved by virtue of tuning over multiple discharges.

Plasma shape control in tokamaks involves basically two steps:

- Identification of the plasma boundary in real time.

- Adjustment of the poloidal field coil currents to bring the real shape as 
close as possible to the preprogrammed shape.

The deployment of the real-time version of the Grad-Shafranov equilibrium reconstruction code LIUQE [16], i.e., RTLIUQE [17], with a sub-ms cycle time (0.4ms), has fulfilled a necessary requirement for the development of a real time plasma position and shape control algorithm, based on the information of poloidal flux and magnetic field provided by the real-time Grad-Shafranov solver. RTLIUQE and other similar real time equilibriums codes [18] provides an approximate solution to the Grad-Shafranov equilibrium relation that yields the best fit to the diagnostic measurements, with additional assumptions on the plasma kinetic profiles, as the diagnostic data are generally insufficient to constrain the problem towards a unique solution. RTLIUQE solutions are produced at a rate that is amply sufficient for discharge control, with the sole exception of the control of the vertical stability on a sub-ms time scale, which is still entrusted to the legacy analogue control system.

The main operational parameters of the Tokamak à Configuration Variable (TCV) [19] are the following: major radius $0.88 \mathrm{~m}$, minor radius $0.25 \mathrm{~m}$, vacuum toroidal field up to $1.5 \mathrm{~T}$, plasma current up to $1 \mathrm{MA}$, elongation up to 2.8 , triangularity from -0.7 to 1 . Figure 1 shows the TCV poloidal cross-section. Two $O H$ coil circuits are used to inductively create the desired toroidal electric field inside the vacuum vessel. The first circuit powers the central solenoid $A 1$; the second circuit powers the coils $B, C$ and $D$ connected in series. The arrangement is designed to minimise the stray fields in the vacuum vessel when the currents in the two circuits are equal. The extreme shaping flexibility is provided by the 16 independently powered poloidal field coils $(E-F)$. The toroidal field in TCV is created by 16 toroidal field coils connected in series. The 16 coils are connected through a bus, modeled with the two poloidal field coils $T 1$ and $T 2$. T3 represents the return loop of the connection. The vertical stabilization of the most highly elongated TCV plasmas is not possible with the main poloidal power supply system and the external poloidal field coils because of the relatively slow switching time of the thyristors and the shielding from the vacuum vessel. Consequently, a fast power supply is used to energise a pair of low-impedance fast internal $G$ coils. The switching frequency is typically $10 \mathrm{kHz}[20$. On TCV, the creation of an extreme variety of plasma shapes and magnetic configuration is accomplished with the help of the Matrix Generation Algorithm and Measurement Simulator (MGAMS) and Free Boundary Tokamak Equilibrium (FBTE) codes [21, 22], the suite of software tools used routinely on TCV to determine the feedforward poloidal coil currents as well as the feedback parameters for a given plasma configuration.

This extensive poloidal-field coil system and the recent successful implementation of RTLIUQE on TCV have paved the way to the design of a new plasma position and shape controller. The Matlab-Simulink environment of the digital control system [23, 24, 25] of TCV provides a flexible platform for implementing new algorithms in parallel with the legacy analogue TCV controller. As a minor but important step along this path, a new controller was also developed to ac- 


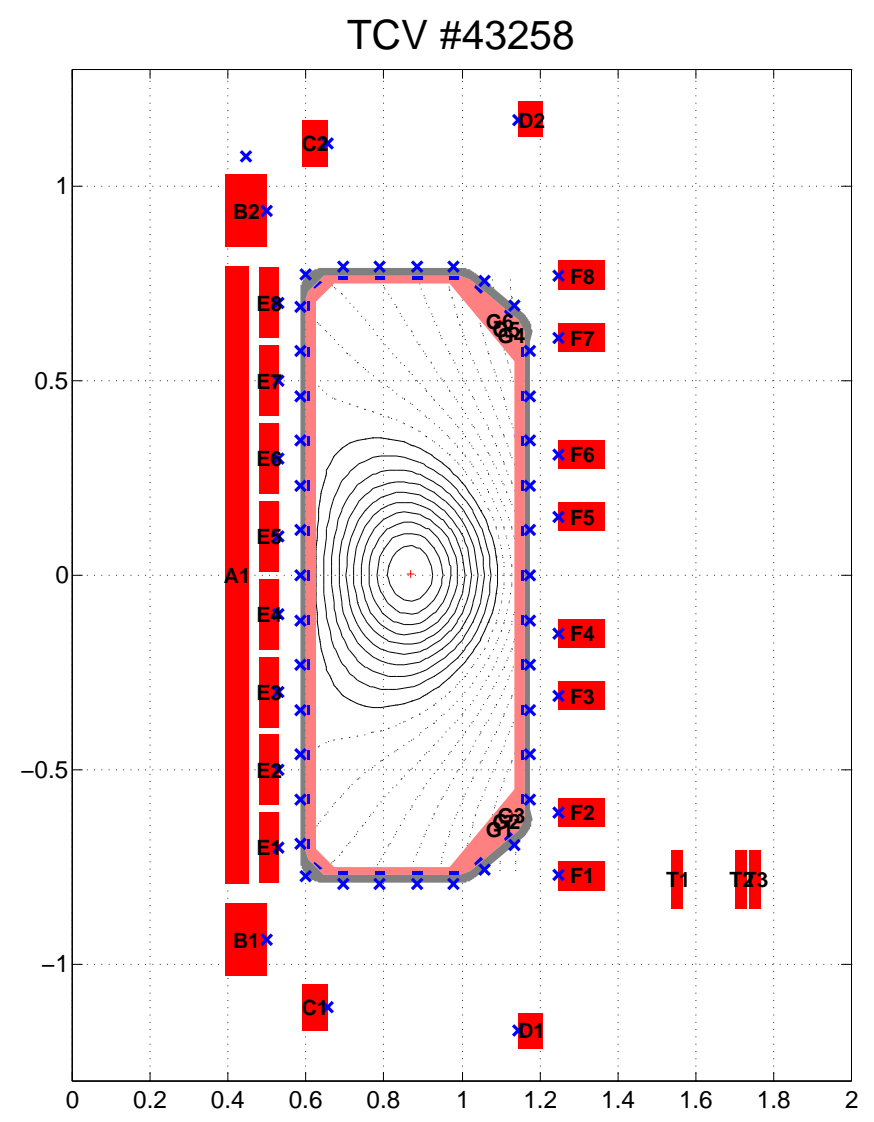

Figure 1: Poloidal cross-section of TCV, showing the flux surfaces obtained from LIUQE, ohmic coils (A-D), the poloidal field coils (E-F), the fast internal coils $(\mathrm{G})$ and the toroidal field coil connections $(\mathrm{T})$.

tuate the sign switching of the poloidal-field coil currents (the so-called 'sign-bit controller').

The paper is structured as follows. Section 2 describes the design and the key features of the new generalised plasma position and shape controller. The formulation of the linearised plasma model of TCV that is used to verify the controller performance and determine the control parameters is reported in section 3. The experimental implementation of the control algorithm on various TCV plasma discharge types is discussed in section 4. A brief overview of the plasma modelling, controller design and experimental results, as well as an outlook for the physics applications of the controller to advanced plasma configurations is provided in section 5. The sign-bit controller is presented in the Appendix 6.4. 


\section{Plasma position and shape controller design.}

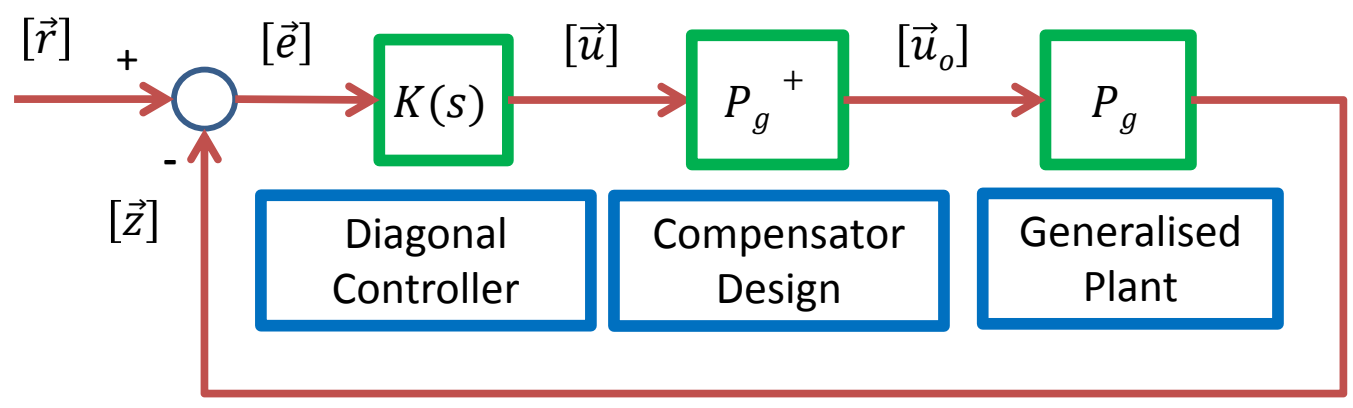

Figure 2: Simplified block diagram representation of plasma shape and position control algorithm.

Different tokamaks employ various approaches to control the plasma position and shape [1, 26, 27]. The generalised plasma position and shape controller is primarily based on the isoflux control scheme. The plasma target shape is defined as a set of control points on the desired plasma boundary, and the poloidal flux at control points is controlled to be equal [18]. For a limiter plasma discharge, the flux differences between adjacent control points are controlled. In the case of divertor discharges, the primary $\mathrm{X}$-point is used as the reference point and the flux error differences between it and the other control points on the plasma boundary are controlled. Similarly, the strike point is controlled by requiring the poloidal flux on the control points on the leg to be equal to that of the $\mathrm{X}$ point [28. X-points are obtained by controlling the poloidal magnetic field at the reference $\mathrm{X}$-point position to zero.

The generalised plasma position and shape controller was developed in two stages: a time-invariant version at first, and then a more general time-varying controller.

\subsection{Controller design}

The architecture of the plant used for the design of the generalised plasma position and shape controller is a MIMO (multiple input and multiple output) system with poloidal field coil currents as inputs and the controlled variables as outputs. A change in one of the inputs will affect all the outputs of the system, that is, there is an interaction between the inputs and the outputs. A specific aim of the design is to convert this to a non-interacting system, where an input only affects its corresponding output. Figure 2 shows the simplified block diagram for the plasma position and shape controller algorithm. A non-diagonal generalised plant $P_{g}$ is defined, where a change in its input would affect all its outputs. Consequently, a compensator design $P_{g}^{+}$, pseudo-inverse of the static 


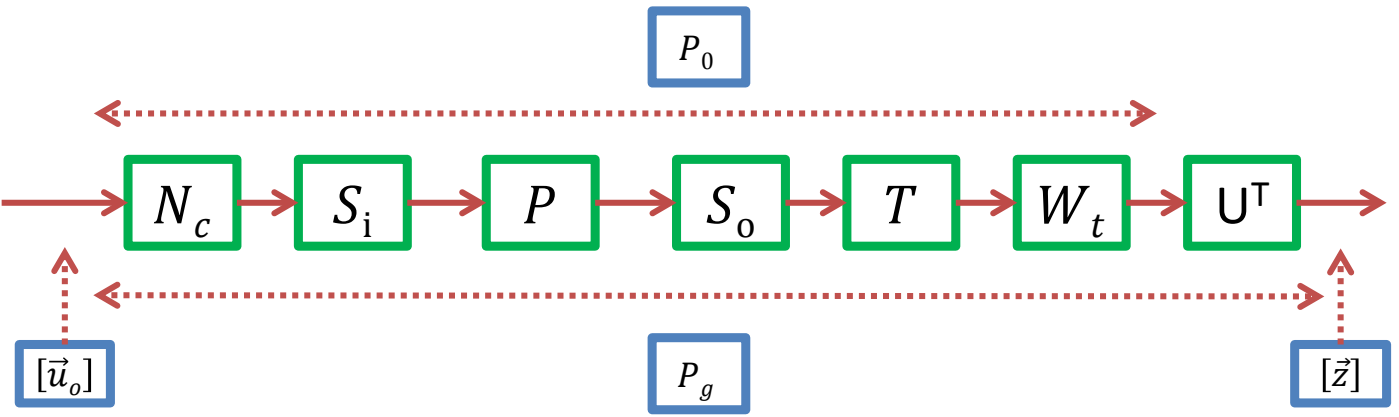

Figure 3: Schematic block diagram for the generalised plant.

(DC) gain of the generalised plant, is derived, provided that $P_{g}$ has a full row (output rank), to counteract the interaction of the plant. The result is a 'newly' shaped plant, $G_{s}=P_{g} P_{g}^{+}$, which is nearly diagonal and easier to control than the original plant $P_{g}$. A diagonal proportional and integral controller $K_{s}$ is utilised, with diagonal gain matrices $K_{s p}$ and $K_{s i}$ to be designed. The control law takes the form of (1), where $\vec{u}$ is the control signal (the vector of 16 poloidal field coil current references) and $\vec{e}=(\vec{r}-\vec{z})$ is the error defined as the difference between the reference $\vec{r}$ and the controlled variables $\vec{z}$.

$$
\begin{gathered}
\vec{u}=K(s) \vec{e} \\
K(s)=K_{s p}\left(1+\frac{K_{s i}}{s}\right)
\end{gathered}
$$

Figure 3 shows the schematic block diagram for the determination of a generalised plant $P_{g}$. A plant $P$ is defined, which accepts poloidal-field coil currents as inputs and outputs the poloidal flux $\overrightarrow{\psi_{b}}$ and poloidal magnetic field $\overrightarrow{B_{b}}$ at the control points. The definition of $P$ is an approximation as it only includes the DC component of a highly dynamic system. In particular, the issue of vertical stability, i.e., the growth of the fast axisymmetric mode, is assumed to be entirely decoupled (e.g., separated in frequency space) from the shape-control problem and to be handled by a separate system (namely, the vertical stability control loop in the legacy hybrid control system). This assumption can never be strictly true, so improvements in controller decoupling are under consideration for the future. Hence, neglecting the dynamics and linearising around an equilibrium point, the plant $P$ with $\delta \vec{I}_{P F}$ as inputs and $\delta \vec{y}$ as outputs is given by (2). Here, $G_{b}$ is the matrix such that $\delta \overrightarrow{\psi_{b}}=G_{b} \delta \vec{I}_{P F}$, where $\delta \vec{\psi}_{b}$ is the poloidal flux at the control points on the plasma boundary, $G_{d}$ is the matrix such that $\delta \vec{\psi}_{d}=G_{d} \delta \vec{I}_{P F}$, where $\delta \overrightarrow{\psi_{d}}$ the poloidal flux at the control points on the divetor leg, $G_{B_{p}}$ is the matrix such that $\delta \overrightarrow{B_{p}}=G_{B_{p}} \delta \vec{I}_{P F}$, where $\delta \overrightarrow{B_{p}}$ is the poloidal magnetic field $(r$ and $z$ compoments) at the X-points. $M_{b}$ is the near-diagonal matrix that trans- 
forms absolute poloidal fluxes to flux differences between points and, similarly, $M_{d}$ is the matrix that transforms absolute fluxes on divertor-leg points to flux differences with respect to the X-point.

$$
P=\left(\begin{array}{c}
M_{b} G_{b} \\
M_{d} G_{d} \\
G_{B_{p}}
\end{array}\right)
$$

The control algorithm includes a constraint, which guarantees that the average addition of poloidal flux at the control points is zero, to prevent interference with the plasma current control. To achieve this, a null space basis $N_{c}$ is determined such that $\left[\mathbf{1}^{T} G_{b}\right] N_{c} u_{o}=0 \forall u_{o} \in \mathbb{R}^{n_{c}-1}$, where $n_{c}$ is the number of poloidal field coils. An input scaling matrix $S_{i}$ ensures common units for the poloidal field coil currents ( $\mathrm{kA}$ in practice) and an output scaling matrix $S_{o}$ scales the plant outputs to dimensionless quantities that are roughly comparable in magnitude.

A crucial element in the design is the matrix $T$, given by (3), which converts the dimensionless plant outputs to physically meaningful estimators, such as,e.g., vertical and radial plasma position estimates, where $\vec{\psi}_{Z}=M_{b} \overrightarrow{d \psi} / d Z / \| M_{b} \overrightarrow{d \psi} / d Z \mid$ and $\vec{\psi}_{R}=M_{b} \overrightarrow{d \psi} / d R /\left\|M_{b} \overrightarrow{d \psi} / d R\right\|$ are the vectors representing the change in the controlled poloidal flux differences due to vertical and radial displacement at the control points on the plasma boundary. A projection of the dimensionless plant outputs onto the vectors corresponding to a vertical and a radial displacement ensures that the first two entries are estimates of the radial/vertical position.

Finally, the estimators are weighted with the help of a weight matrix $W_{t}$, which provides a means to prioritise the various meaningful plasma estimators based on their level of importance for a given plasma configuration.

$$
T=\left(\begin{array}{ccc}
\vec{\psi}_{Z}^{T} & 0 & 0 \\
\vec{\psi}_{R}^{T} & 0 & 0 \\
I & 0 & 0 \\
0 & I & 0 \\
0 & 0 & I
\end{array}\right),
$$

A new plant $P_{o}=W_{t} T S_{o} P S_{i} N_{c}$ is thus finally defined. The next step is to perform a singular value decomposition (SVD) [29] of $P_{o}=U \Sigma V^{T}$, which provides a matrix $V$, representing a set of orthogonal vectors in the admissible input coil current space and $U$, the output directions. This organises the controlled variables into large and small singular values represented by (4), and as a result provides the freedom of limiting the control to the $n_{1}$ 'largest' singular values of $\Sigma_{1}$, obtained by projecting the weighted errors on the output space of these singular values, $U_{1}$.

$$
P_{o}=\left(\begin{array}{ll}
U_{1} & U_{2}
\end{array}\right)\left(\begin{array}{ccc}
\Sigma_{1} & 0 & 0 \\
0 & \Sigma_{2} & 0
\end{array}\right)\left(\begin{array}{c}
V_{1}^{T} \\
V_{2}^{T} \\
V_{3}^{T}
\end{array}\right)
$$


The generalised plant $P_{g}$ is then defined as $P_{g}=U^{T} P_{o}$. The controller for the original plant $P$ is thus represented by (5).

$$
C_{p}(s)=S_{i} N_{c} P_{g}^{+} K(s)
$$

The controller designed above is only applicable to plasma scenarios involving a plasma shape and position that remain fixed during the discharge (time invariant controller design). The next step is to tackle time-varying configurations. The chosen approach is to construct individual controllers for the equilibria predefined by the shot design sequence. These are, however, sampled at a smaller time step than the original sample times. A linear interpolation in time is performed between the control points defined by various equilibria during the shot design phase. The sample time of the linear interpolation is usually chosen as $10 \mathrm{~ms}$. The individual controllers with respect to each of the interpolated equilibria at smaller time steps are then determined using (5). Measures are taken such that the output of the currently active controller $C_{p}^{i}(s)$ switches to the output of the subsequent controller $C_{p}^{i+1}(s)$ smoothly ensuring a continuous transition between the controllers throughout the plasma discharge, where $i$ represents the index of the controller (Time varying controller design). The technique for smoothly switching between the controllers is presented in the Appendix 6.1. In addition, techniques for handling the saturation in the poloidal field coil currents and the discontinuity introduced by the switching between the position control of the TCV hybrid controller to the position control performed by the designed controller is presented in Appendix 6.2.

\subsection{Implementation with the TCV hybrid controller}

Figure 4 shows a block diagram representation of the TCV hybrid controller with the generalised plasma position and shape controller. The simplified scheme of the TCV hybrid controller is shown in the grey box of figure 4. The measurements (magnetic flux loops, magnetic probes, FIR laser interferometer fringe counter, coil current measurements) from the TCV tokamak are processed by the $A$ matrix, generating controlled variables, as linear combinations of the inputs signals. The set of controlled variables consists of the plasma current $I_{p}$, the poloidal field coil currents $I_{p f}$, the vertical position estimator $z I_{p}$ and the radial position estimator. The controlled variables are then subtracted from the reference signals to yield the respective error signals. The error signals are fed to their respective controllers and actuators: $C_{I p}(s)$ represents a proportional and integral controller and $T_{I p}$ selects the ohmic coils as an actuator for controlling the plasma current; $C_{Z}(s)$ represents a proportional and differential controller and $T_{z}$ selects a combination of the $F$ poloidal field coils to control the plasma vertical position; $C_{R}(s)$ is a proportional controller and $T_{R}$ selects a combination of the $F$ poloidal field coils to control the radial position of the plasma; $C_{F P S}(s)$ is a differential controller and $T_{F P S}$ selects the internal $\mathrm{G}$ coils to control the plasma vertical instability; $C_{I p f}(s)$ is a proportional controller and $T_{I p f}$ selects 


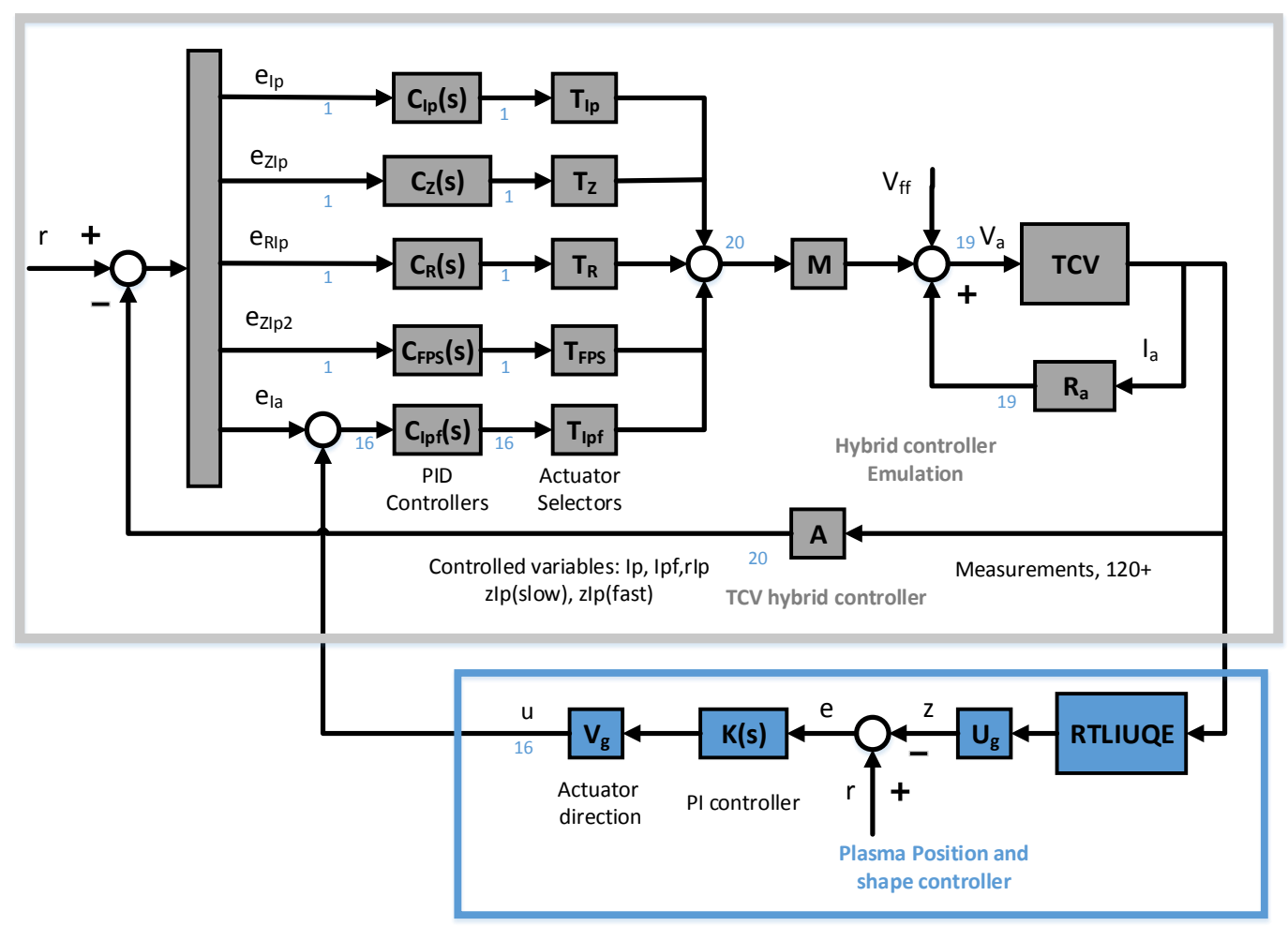

Figure 4: Block diagram showing the implementation of the generalised plasma position and shape controller with the TCV hybrid controller.

the combination of poloidal field coils orthogonal to the coil combination providing vertical and radial control. The actuator control signals are then passed through the $M$ matrix which is constructed with mutual inductance coefficients, i.e., performs the decoupling of the mutual inductances. The resistive compensation and the feedforward voltages $V_{f f}$ are added to the outputs of the $M$ matrix to generate the input voltages $V_{a}$ for the actuators.

The implementation of the generalised plasma position and shape controller with the TCV hybrid controller is shown in the blue box of figure 4][30]. The controller algorithm resides on the same real-time node as the TCV hybrid controller and functions on a cycle time of $0.1 \mathrm{~ms}$. The controller formalism defined in the section 2 relies on the legacy analogue TCV hybrid controller for stabilizing the vertical position of the plasma [31], that is, only the differential control in the vertical control loop remains in the TCV hybrid controller. To ensure a reliable radial and vertical position control of the plasma, an appropriate weighting is applied on the position estimators in the generalised plasma position and shape controller such that the control of the first two controlled variables corresponds to plasma position control. The isoflux surfaces generated by the poloidal field coil directions corresponding to the first two controlled variable controlling the vertical and the radial plasma position, are shown in figure 5. The RTLIUQE 
provides the poloidal flux and the poloidal magnetic field at the control points on the plasma boundary. The controller design includes a pre multiplier matrix $U_{g}=U^{T} W_{t} T S_{o}$ and a post multiplier matrix $V_{g}=S_{i} N_{c} P_{g}^{+}$: the latter translates the RTLIUQE outputs to controlled variables, $\vec{z}$, while the former provides the coil directions for controlling the controlled variables. The reference signals are defined as zeros. The error signals are fed to a proportional and integral controller $K(s)$ defined by (1). The control algorithm is developed in Matlab-Simulink environment and the Simulink code is then used to study the performance of the controller with the plasma model described in section 3. The Simulink code was later implemented in the digital control system of TCV to minimise coding errors and development time.
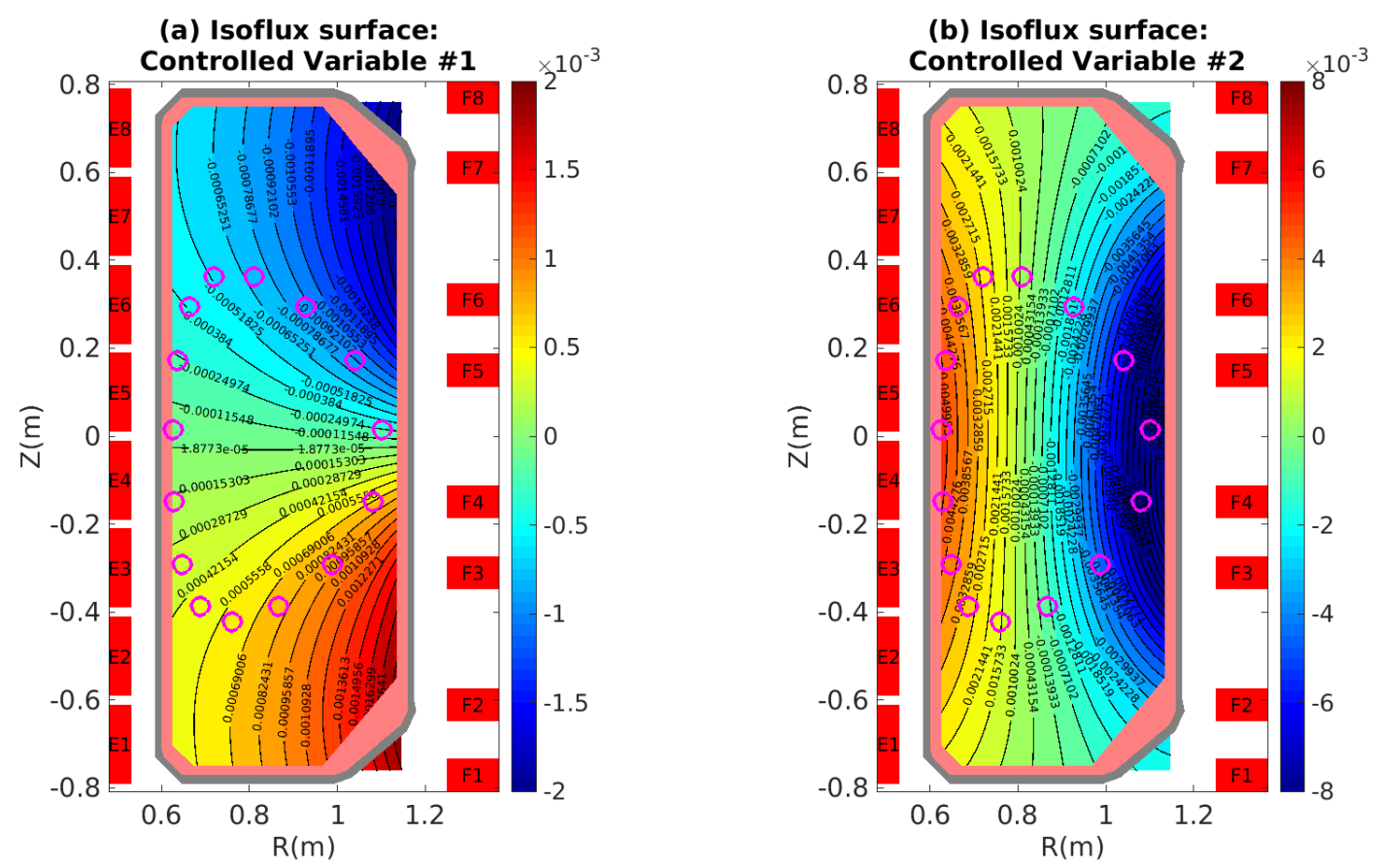

Figure 5: Isoflux surfaces generated by the poloidal field coils $E 1-E 8$ and $F 1-F 8$ for a limiter plasma configuration for controlling the (a) first and (b) second controlled variable. The plasma boundary is denoted by the magenta circles.

\section{Plasma Modelling}

The optimization of the control parameters for the generalised plasma position and shape control is performed with the help of a linearised plasma response model, the RZIP model [32]. Although various assessed and validated linearised plasma models are present [33], due to the assumption that plasma shape control problem lies in the low frequency range, allows the use of a simplified RZIp 
plasma model for tuning and verifying the control algorithm. The model is constructed with physical laws and simplifying assumptions. A set of circuit equations is developed, based on the supposition that the plasma current distribution remains constant during any control action, but that its centroid can move vertically and radially and its integral, the total plasma current, can change. The plasma model is based on the linearization assumption that small variations in the poloidal field coil voltages lead to small changes in the plasma current, poloidal field currents, vacuum vessel currents and plasma radial and vertical positions, about a given unperturbed equilibrium state.

The set of equations composing the model comprises the circuit equations for the poloidal field coils, the vessel currents and the plasma, in addition to the plasma radial and vertical force balance equations. All spatially dependent quantities are evaluated using the plasma current distribution derived by the LIUQE equilibrium code,

$$
\langle A\rangle=\frac{\sum_{i} A\left(R_{i}, Z_{i}\right) J_{\phi}\left(R_{i}, Z_{i}\right)}{\sum_{i} J_{\phi}\left(R_{i}, Z_{i}\right)}
$$

where A is any parameter of interest.

Linearisation of the set of equations around an equilibrium and defining $M$, $R, \vec{x}(t)$ and $\vec{u}(t)$ as follows,

$$
\begin{gathered}
M=\left(\begin{array}{ccccc}
M_{a a} & M_{a v} & M_{a p_{o}} & \frac{\partial M_{a p}}{\partial R} & \frac{\partial M_{a p}}{\partial Z} \\
M_{v a} & M_{v v} & M_{v p_{o}} & \frac{\partial M_{v p}}{\partial R} & \frac{\partial M_{v p}}{\partial Z} \\
M_{p a_{o}} & M_{p v_{o}} & L_{p_{o}} & M_{p R} & 0 \\
\frac{\partial M_{p a}}{\partial R} & \frac{\partial M_{p v}}{\partial R} & M_{R p} & M_{R R} & M_{R Z} \\
\frac{\partial M_{p a}}{\partial Z} & \frac{\partial M_{p v}}{\partial Z} & 0 & M_{Z R} & M_{Z Z}
\end{array}\right), R=\left(\begin{array}{ccccc}
R_{a} & 0 & 0 & 0 & 0 \\
0 & R_{v v} & 0 & 0 & 0 \\
0 & 0 & R_{p_{o}} & R_{p R} & 0 \\
R_{R a} & R_{R v} & R_{R p} & R_{R R} & 0 \\
R_{Z a} & R_{Z v} & 0 & 0 & 0
\end{array}\right) \\
\vec{x}(t)=\left(\begin{array}{c}
\delta I_{a} \\
\delta I_{v} \\
\delta I_{p} \\
I_{p o} \delta R \\
I_{p o} \delta Z
\end{array}\right), \vec{u}(t)=\left(\begin{array}{c}
\delta V_{a} \\
0 \\
0 \\
S_{R} \\
0
\end{array}\right)
\end{gathered}
$$

where the set of equations and the elements of the matrices $M$ and $R$ are defined in the appendix 6.3 .

The complete dynamic system including the conductors and plasma can be expressed in the state space form with $\vec{u}$ as inputs and $\vec{y}$ as outputs of the system

$$
\begin{aligned}
& \overrightarrow{\dot{x}}=A \vec{x}+B \vec{u} \\
& \vec{y}=C \vec{x}+D \vec{u}
\end{aligned}
$$

where the first equation is called the state equation with $A=-M^{-1} R$ and $B=M^{-1}$, respectively. and the second equation is called the output equation. The only positive eigenvalue of matrix $A$ is the plasma vertical displacement event growth rate which is a specific characteristic of the plasma's vertical speed 
of response. For typical discharge shapes of TCV, the vertical growth rate is about $200-300 s^{-1} \cdot \vec{y}$ represent a vector of the measurements consisting of the radial $r I_{p}$ and vertical $z I_{p}$ estimators of the TCV hybrid controller and the controlled variables $\vec{z}$ of the generalised plasma position and shape controller. $C$ is a matrix relating the measurement to the states of the system and $D$ is a direct feedthrough matrix denoting the sensitivity of the measurements to the inputs and are represented as follows

$$
C=\left(\begin{array}{l}
C_{r I_{p}} \\
C_{z I_{p}} \\
C_{z}
\end{array}\right), D=0
$$

The matrices $C_{r I_{p}}$ and $C_{z I_{p}}$ converts the states to plasma position estimators of the TCV hybrid controller and matrix $C_{z}$ coverts the states into the controlled variables of the generalised plasma position and shape controller.

The loop for the reconstructed radial, $r I_{p}$ and vertical, $z I_{p}$ position estimators from the RZIP plasma model is closed with the position controller from the TCV hybrid controller described in section 2.2. A stable closed loop step response is shown in figure 6(a) for the vertical position estimator for the TCV tokamak for a given proportional $K_{p}$ and differential control $K_{d}$ in the TCV hybrid controller.

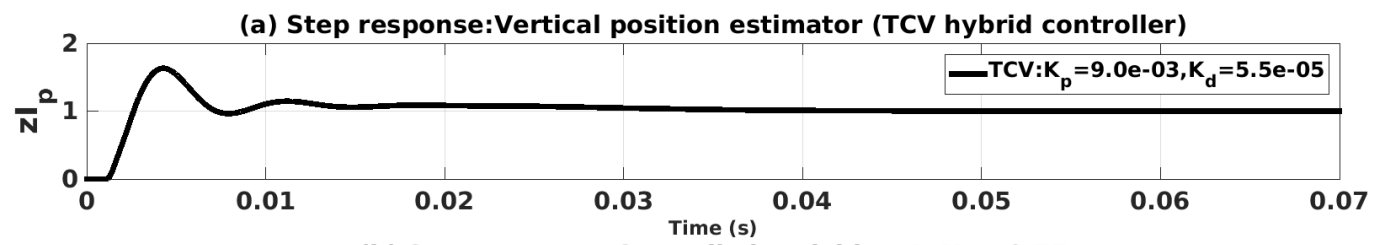

(b) Step response:Controlled variable \#1, $\mathrm{K}_{\mathrm{sp}}=\mathbf{0 . 5 5}$

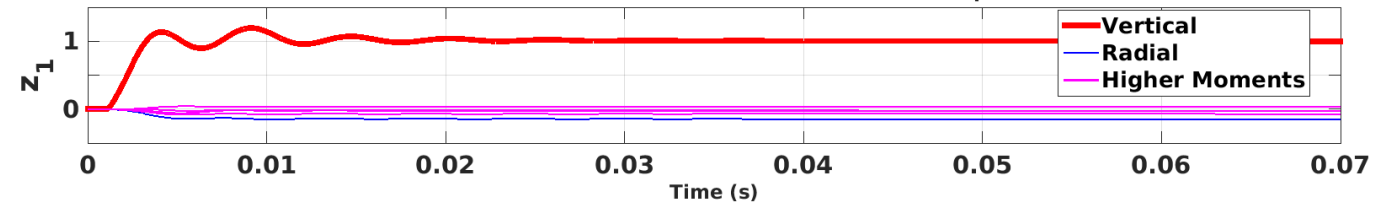

(c) Step response:Controlled Variables \#1,\#2,\#3

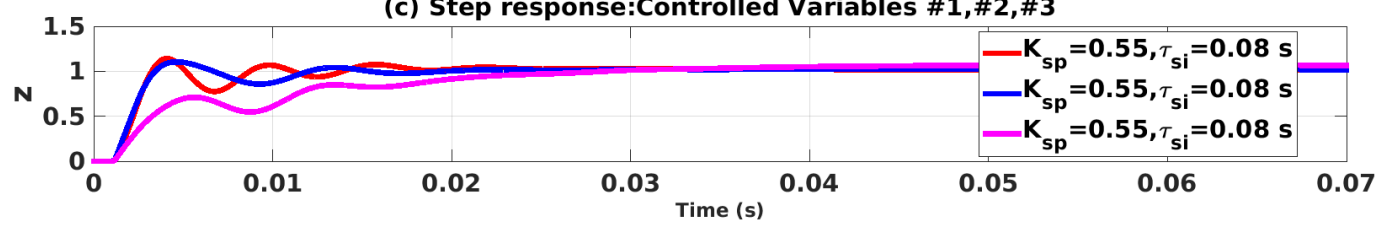

Figure 6: Linearised plasma modelling simulation results for the TCV tokamak. (a) Step response of the vertical estimator for the TCV hybrid controller and (b) step response of the first controlled variable and its effect on the higher order controlled variables for the generalised plasma position and shape controller. (c) Step response of the controlled variables controlling the plasma position and shape.

A stable response with respect to a step reference on the first controlled variable of the generalised plasma position and shape controller derived from 
the RZIP plasma model is shown in figure 6(b) with only a proportional gain $K_{s p}$. For a given plasma current distribution and set of controller gains, the ratio between the open loop gain for the generalised plasma position and shape controller and that for the position controller within the TCV hybrid controller from the simulation is found to be 1.5. Thus, for the given case, the simulation predicts the use of either a higher differential or a lower proportional gain within generalised plasma position and shape controller in order to have a comparable response. In general, the modelling predicts that the differential gain in the TCV hybrid controller or the gains of the new controller are to be modified in order to obtain an adequate performance.

The RZIp simulation not only predicts the fact that a simple proportional control on the first controlled variable related to the vertical position of the plasma can provide a suitable controller performance, but also shows the coupling among the controlled variables in steady state. Figure 6(b) shows finite steady state offsets in the response of the remaining controlled variables with respect to a step reference on the first controlled variable. A comparison between the step response on the first two controlled variables (related to the plasma position control) and a higher order controlled variable (related to the control of the plasma shape) for a fixed proportional $K_{s p}$ and integral gain $K_{s i}=1 / \tau_{s i}$ is shown in figure 6(c). A slower dynamical response of the higher order controlled variable in comparison to the first two is observed for a fixed proportional and integral gain on the controlled variables, pointing towards higher gains on the the higher order controlled variables to improve the tracking performance of the plasma shape.

\section{Experimental results}

Dedicated experiments have been performed to test the generalised plasma position and shape controller during the TCV campaign in 2016-2017. The analysis of the experimental results is divided into two main sections, with each section comprising of application of the controller design on plasma scenarios including fixed as well as time varying plasma position and shape.

\subsection{Limiter plasma configuration}

\subsubsection{Plasma configuration with fixed plasma shape and position}

Limiter plasma discharges (54105 and 54111) involving fixed plasma position and shape in time were chosen to test the controller formalism with the aim of exploring the range of proportional and integral gain for controlling the plasma position. In the discharge 54105, the proportional gain of the first two controlled variable representing the control of the plasma position was scanned while setting the integral gain for all controlled variables to 0. Similarly, in the discharge 54111, 
the integral gain on the first two controlled variables was scanned with a fixed proportional gain in the controller.
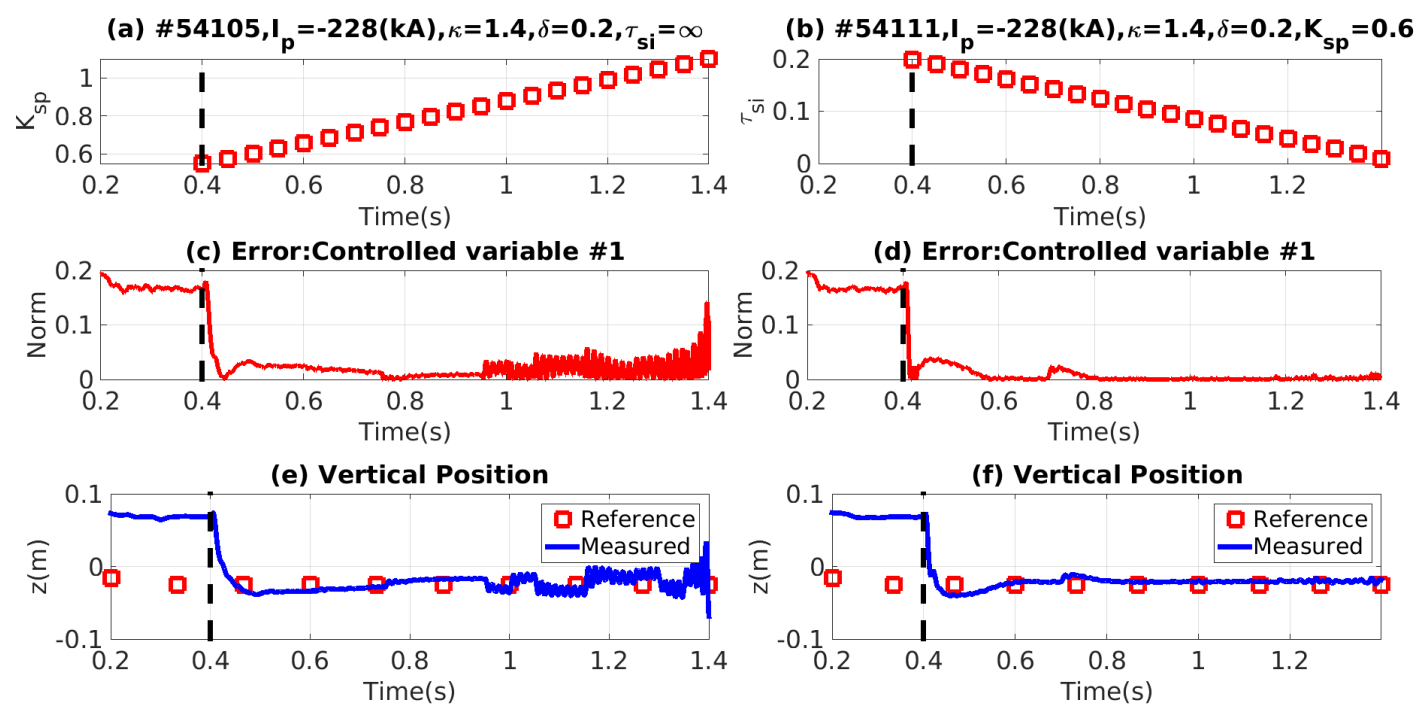

Figure 7: Investigation of the effect of proportional and integral gains on the performance of the generalised plasma position and shape controller. (a) Proportional gain scan without integral gain and (b) integral gain scan with a fixed proportional gain for a limiter plasma configuration with fixed plasma shape and position. (c) and (d) Time evolution of the norm of the error on the first controlled variable related to the plasma vertical position. (e) and (f) Reference and the measurement of the vertical position of the plasma magnetic axis obtained from RTLIUQE. $\delta$ and $\kappa$ represents the reference of the plasma triangularity and elongation from FBTE.

Figure 7 illustrates the range of control parameters, i.e. proportional and integral gains, that provide stability in conjunction with the differential vertical controller of the TCV hybrid controller. Figures 7(a) and 7(b) show a median range of consistent proportional (0.55-0.85) and integral gains (0.02-0.2 s) for a given limiter plasma configuration. Insufficient gains are usually identified experimentally by loss of control, excessive gains by oscillatory behaviour. (figures 7 (c) and $7(\mathrm{~d}))$. Figures $7(\mathrm{e})$ and $7(\mathrm{f})$ show the associated vertical position reference and measurement for the given discharges.

For validating the feature of the controller design of limiting the controlled variables to the set that is most easily controlled, while respecting the hardware limits on the poloidal field coil currents, a series of limiter plasma discharges with fixed plasma shape and position were performed for a given set of controller gains $\left(K_{s p}=0.55, \tau_{s i}=0.2 s\right)$, every successive discharge including a larger set of active controlled variables. Figure 8 explores the effective number of active controlled variables that are required to provide a suitable control of plasma position and shape while limiting the demand on the poloidal field coil currents. Figures 8 (c) and 8 (d) show the norm of the normalised poloidal field coil currents 

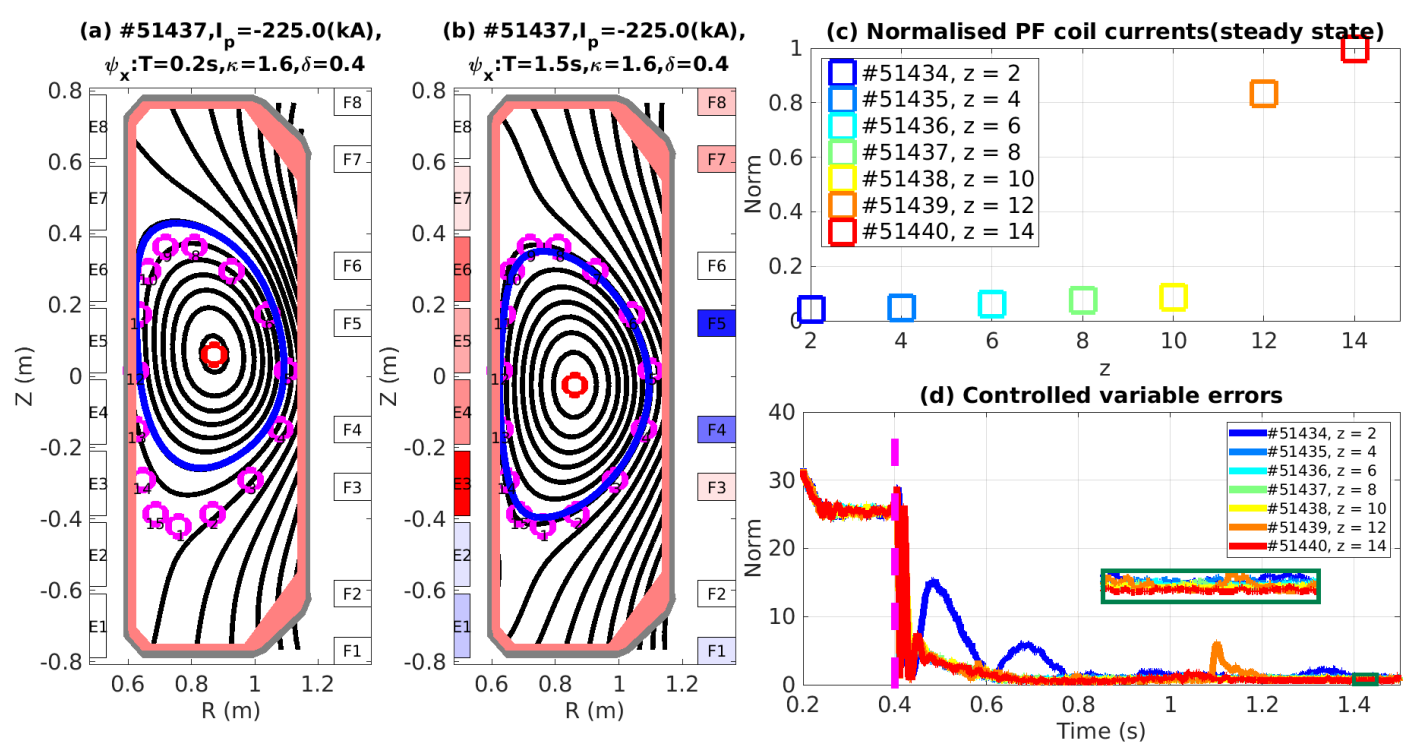

Figure 8: Investigation of optimum number of actively controlled variables for plasma position and shape control for a limiter plasma configuration with a fixed set of controller gains $\left(K_{s p}=0.55, \tau_{s i}=0.2 s\right)$. (a) Poloidal flux map (black lines) $\psi_{x}$ and (b) last closed flux surface (blue line) obtained from RTLIUQE before and after the controller activation with an optimum number of active controlled variables (8). (c) Norm of the normalised poloidal field coil currents requested by the controller at steady state and (d) norm of the error of all the controlled variables with time as a function of the actively controlled variables. The preprogrammed control points are given by the magenta circles. $\delta$ and $\kappa$ represents the reference of the plasma triangularity and elongation from FBTE.

requested by the controller and the norm of the errors on the full set of controlled variables as functions of the number of actively controlled variables. These plots demonstrate that controlling a subset (with high singular values) of variables is sufficient to provide satisfactory shape and position control, and conversely that there is little to gain in adding control channels that have small singular values - rather, this increases the danger that coil current limits are violated. Figures $8(\mathrm{a})$ and $8(\mathrm{~b})$ show the contour plots of the poloidal flux distribution and the last closed flux surface (LCFS), with the control points defining the plasma boundary, at time instants before and after the controller activation for the discharge 51437 with 8 actively controlled variables and fixed set of controller gains $\left(K_{s p}=0.55, \tau_{s i}=0.2 s\right)$. They illustrate the enhancement in the plasma position and shape in comparison to the TCV hybrid controller for a given set of optimised control parameters and a fixed number of actively controlled variables.

\subsubsection{Plasma configuration with varying plasma shape and position}

Two limiter plasma discharges were performed, one containing a scan of the plasma vertical position, followed by a scan in the plasma elongation (55141) and 

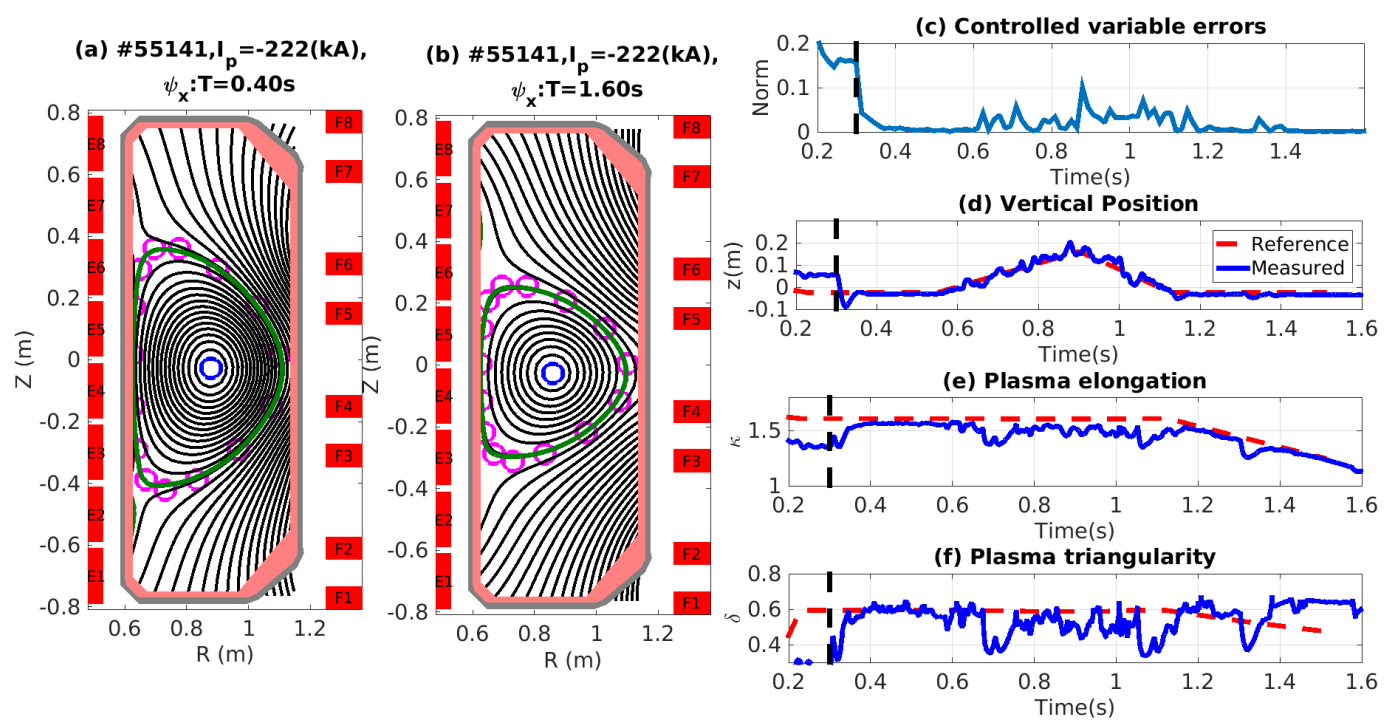

Figure 9: Performance of the generalised plasma position and shape controller for limiter plasma configuration involving a scan of plasma vertical position and elongation with 8 actively controlled variables and fixed controller gains for position $\left(K_{s p}=0.55\right.$ and $\left.\tau_{s i}=0.1 s\right)$ and shape control $\left(K_{s p}=0.3\right.$ and $\left.\tau_{s i}=0.01 s\right)$. (a) Poloidal flux contour $\psi_{x}$ (black lines) and (b) the last closed flux surface (green line) at different time instants of the controller operation obtained from RTLIUQE. (c) Time evolution of norm of the errors of the actively controlled variables. Reference and measurement of the plasma vertical position (d), elongation (e) and triangularity (f) obtained from FBTE and LIUQE. The pre-programmed plasma boundary is given by control points in magenta circles.

the other including a scan from negative to positive plasma triangularity (55144) to test the performance of the time varying version of the controller design with an optimised number of actively controlled variables (8) and controller gains (position $\left(K_{s p}=0.55\right.$ and $\left.\tau_{s i}=0.1 s\right)$ and shape control $\left(K_{s p}=0.3\right.$ and $\tau_{s i}=$ $0.01 s))$.

Figure 9 shows the application of the controller to the complex plasma scenario involving sweeps of plasma position and elongation. The norm of the errors on the active controlled variables (figures 9 (c) are reduced rapidly at the time of the controller activation and are then kept close to zero, providing a satisfactory tracking of the plasma position $(9(\mathrm{~d})$ ) and shape variables (elongation (figure 9(e)) and triangularity (figures 9(f)). Figure 10 shows the evolution of the last closed flux surface at various time instances during the discharge.

Figure 11] shows the application of the controller to a scenario involving a scan of the plasma triangularity from a negative to a positive value. Upon controller activation, the norm of the errors on the actively controlled variables is reduced and adequate tracking of not only the plasma triangularity (figure 11(f)) but also of the plasma vertical position (figure 11(d)) and elongation (figure 11(e)) is obtained. The time evolution of the last closed flux surface during the discharge 

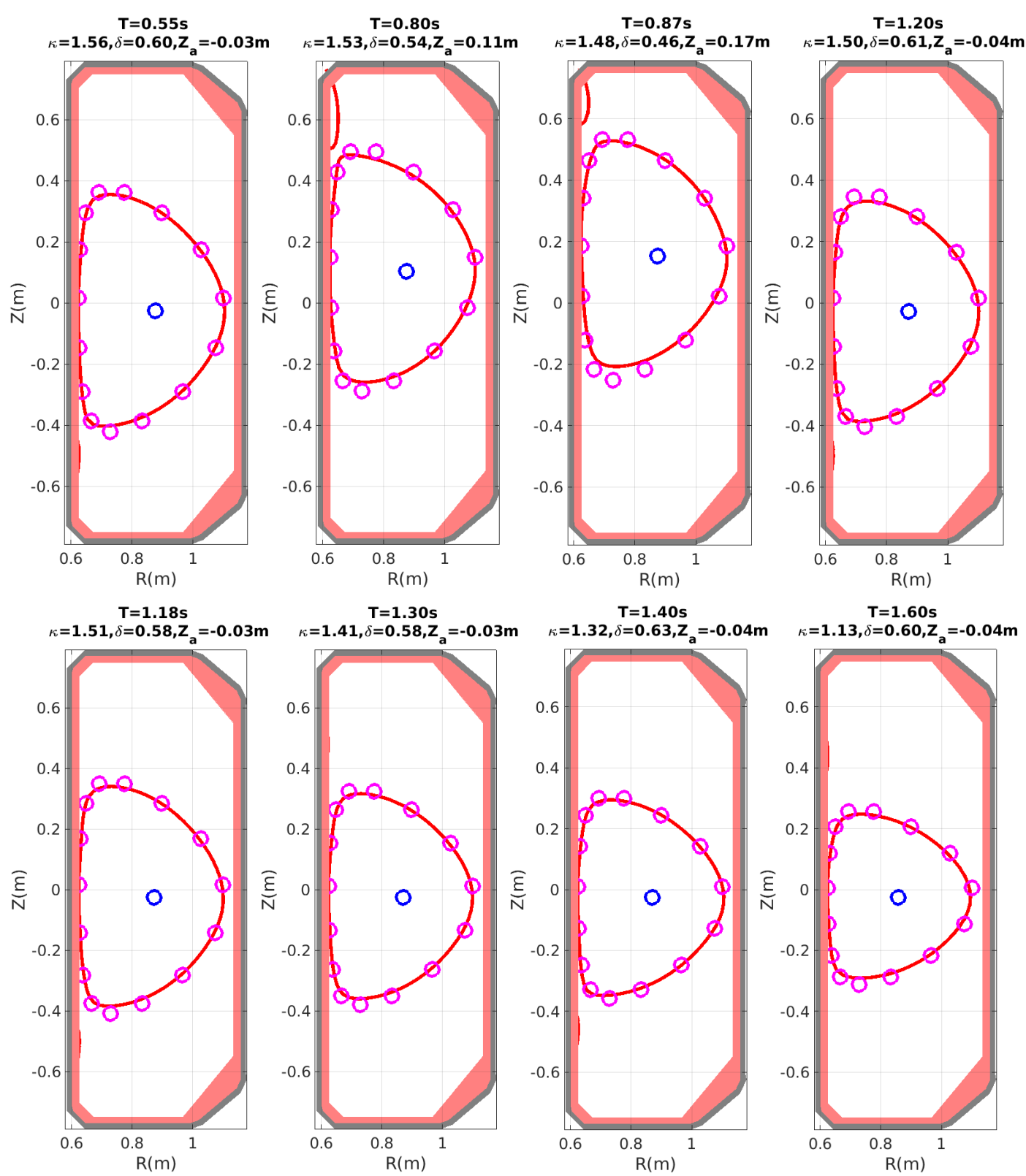

Figure 10: Last closed flux surface (red line) and vertical position $Z_{a}$ of the plasma magnetic axis (blue circle) obtained from RTLIUQE for the plasma discharge 55141, at various time instances. $\kappa$ and $\delta$ represent the plasma elongation and triangularity obtained from LIUQE. The pre-programmed plasma boundary is denoted by magenta circles. 

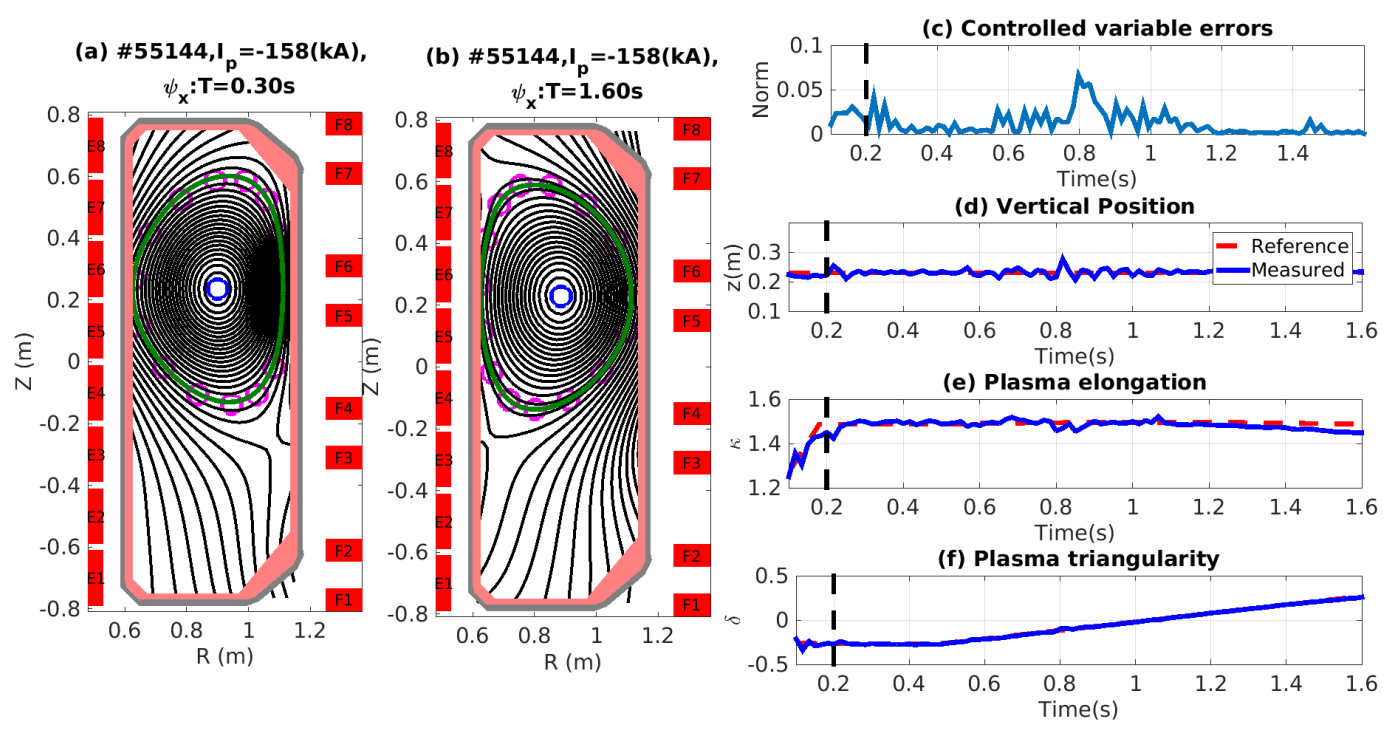

Figure 11: Performance of the generalised plasma position and shape controller for a limiter plasma configuration involving a scan of the plasma triangularity with 8 actively controlled variables and fixed controller gains for position $\left(K_{s p}=\right.$ 0.55 and $\left.\tau_{s i}=0.1 s\right)$ and shape control $\left(K_{s p}=0.3\right.$ and $\left.\tau_{s i}=0.01 s\right)$. (a) Poloidal flux contour $\psi_{x}$ (black lines) and (b) last closed flux surface (green line) at different time instants of the controller operation, obtained from RTLIUQE. (c) Time evolution of norm of the errors of the actively controlled variables. Reference and measurement of the plasma vertical position (d), elongation (e) and triangularity (f) obtained from FBTE and LIUQE. The pre-programmed plasma boundary is given by control points in magenta circles.

is shown in figure 12 .

The perturbations observed in the norm (figures $9(\mathrm{c})$ and 11(c)) of the errors on the controlled variables are induced by step changes in the poloidal field coil currents resulting from a change in polarity or from switching of the controller algorithm to control a new equilibrium. However, the designed controller rejects the disturbances and forces the controlled variables to track the desired reference.

\subsection{Divertor plasma configuration}

\subsubsection{Plasma configuration with fixed plasma shape and position}

After successfully testing the controller design for fixed and time varying plasma position and shape for the limiter plasma configuration, the controller design was extended to divertor plasma configurations. Similar to the previously discussed procedure, a divertor discharge with fixed plasma shape and position was performed (55725). Figure 13 shows the successful application of the controller to a time invariant divertor plasma configuration with an optimised set of controlled variables controlling simultaneously not only the plasma shape and position but 

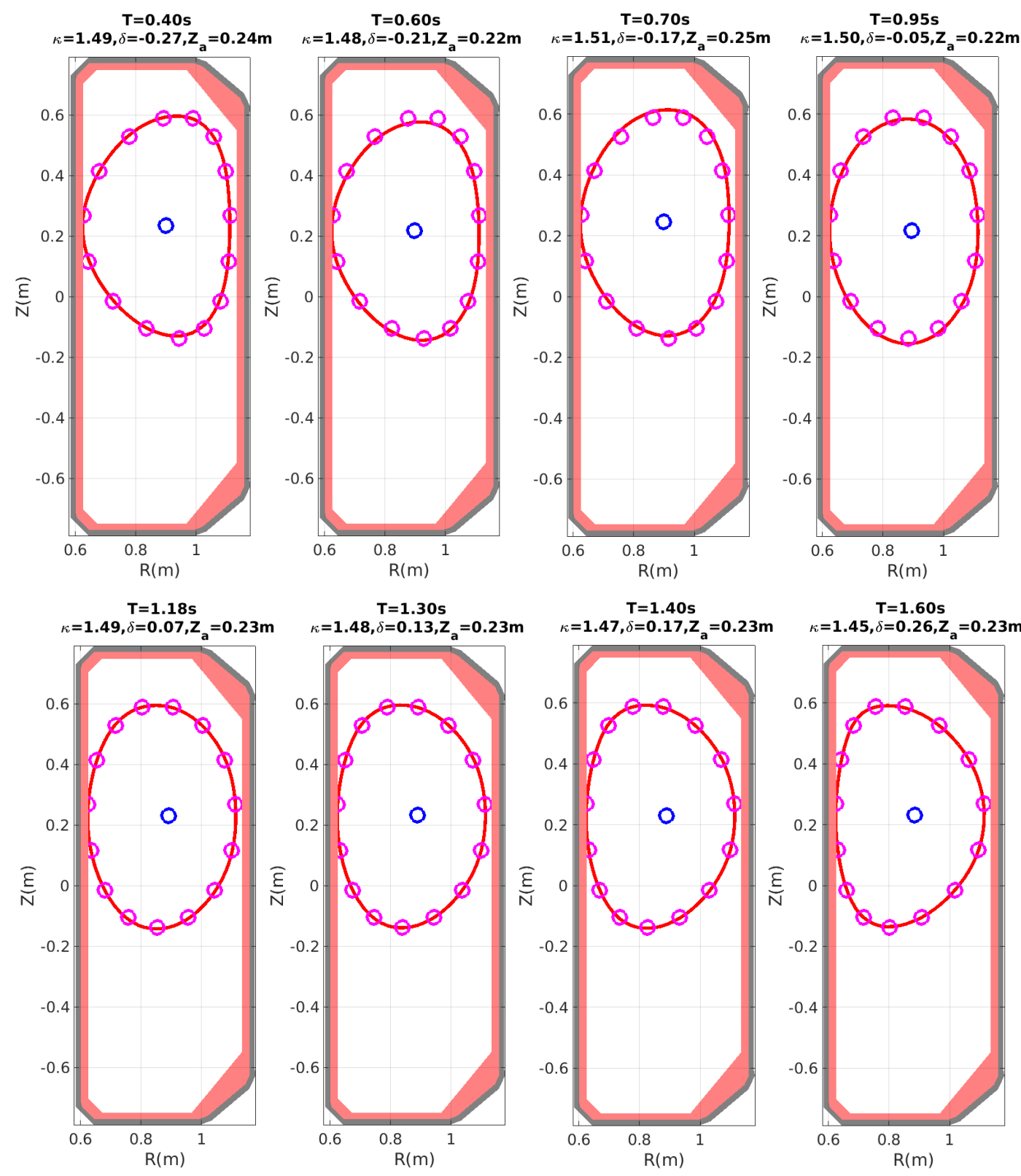

Figure 12: Last closed flux surface (red line) and vertical position $Z_{a}$ of the plasma magnetic axis (blue circle) obtained from RTLIUQE for the plasma discharge 55144, at various time instances. $\kappa$ and $\delta$ represent the plasma elongation and triangularity obtained from LIUQE. The pre-programmed plasma boundary is denoted by magenta circles. 
also the position of the divertor leg and the poloidal magnetic field at the Xpoint. The contour plots before and after the controller activation are shown in figures 13(a) and 13(b). Again, the controller activation results in the reduction of the norm of the errors on the actively controlled variable (figure 13(c)), and excellent and smooth tracking is achieved as shown by a comparison of the references with the measurements of the plasma position and shape variables, as shown in figure $13(\mathrm{~d})$ and figures $13(\mathrm{e}-\mathrm{f})$.

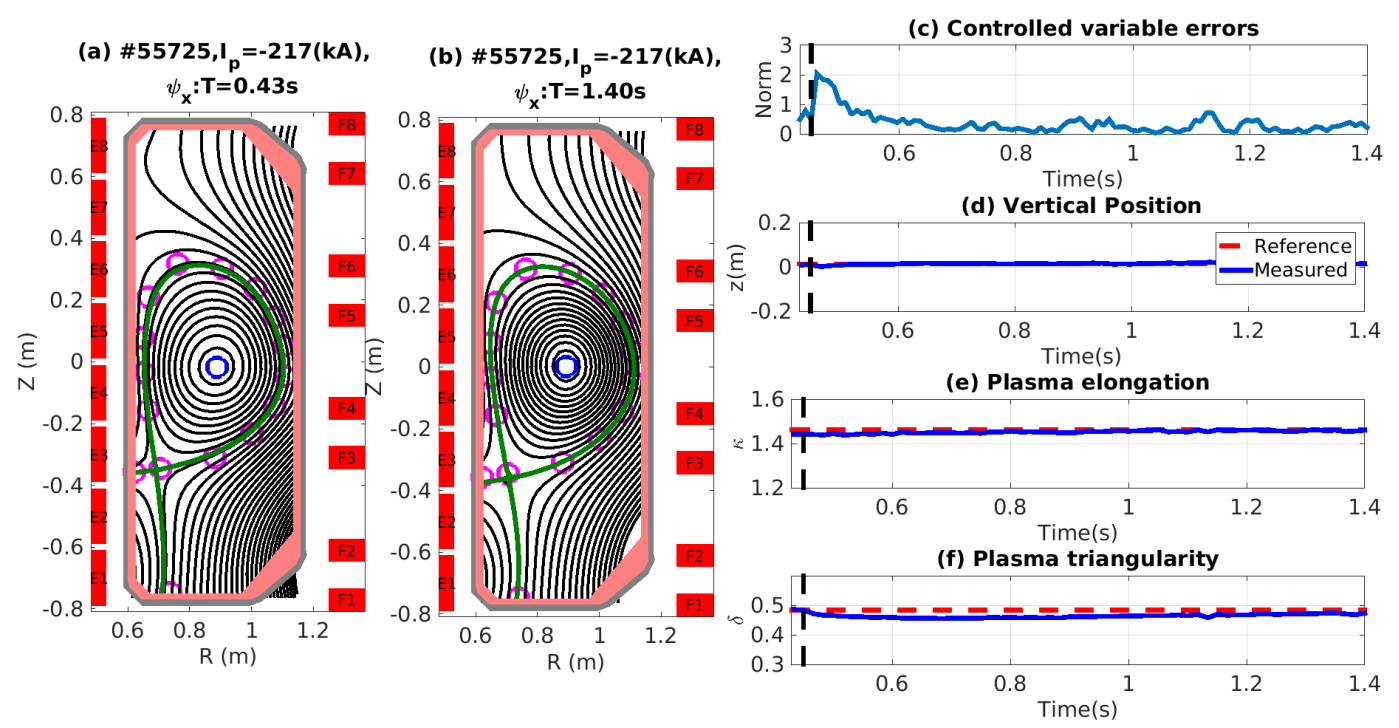

Figure 13: Performance of the generalised plasma position and shape controller for controlling a time varying divertor plasma configuration with optimised actively controlled variables (8) and controller gains for position $\left(K_{s p}=0.55\right.$ and $\left.\tau_{s i}=0.1 s\right)$ and shape control $\left(K_{s p}=0.85\right.$ and $\left.\tau_{s i}=0.05 s\right)$. (a) Poloidal flux contour $\psi_{x}$ (black lines) and (b) last closed flux surface (green line) at different time instants of the controller operation, obtained from RTLIUQE. (c) Time evolution of norm of the errors of the actively controlled variables. Reference and measurement of the plasma vertical position $(\mathrm{d})$, elongation (e) and triangularity (f) obtained from FBTE and LIUQE. The pre-programmed plasma boundary is given by control points in magenta circles.

\subsubsection{Plasma configuration with varying plasma shape and position}

A complex divertor plasma discharge to test the time varying version of the algorithm in a divertor configuration was performed (55147) including sweeps in both the plasma and shape variables. Figure 14 illustrates the universality and flexibility of the controller using a divertor plasma configuration involving complex and simultaneous changes in the plasma position and shape during the plasma discharge. Figure 14 (c) shows the norm of the errors on the actively controlled variables. The tracking performance of the controller with respect to the plasma position and shape variables is shown in figures $14(\mathrm{~d})$ and $14(\mathrm{e}-\mathrm{f})$. 
Figure 15 shows the evolution of the separatrix at various time instances for the plasma discharge.
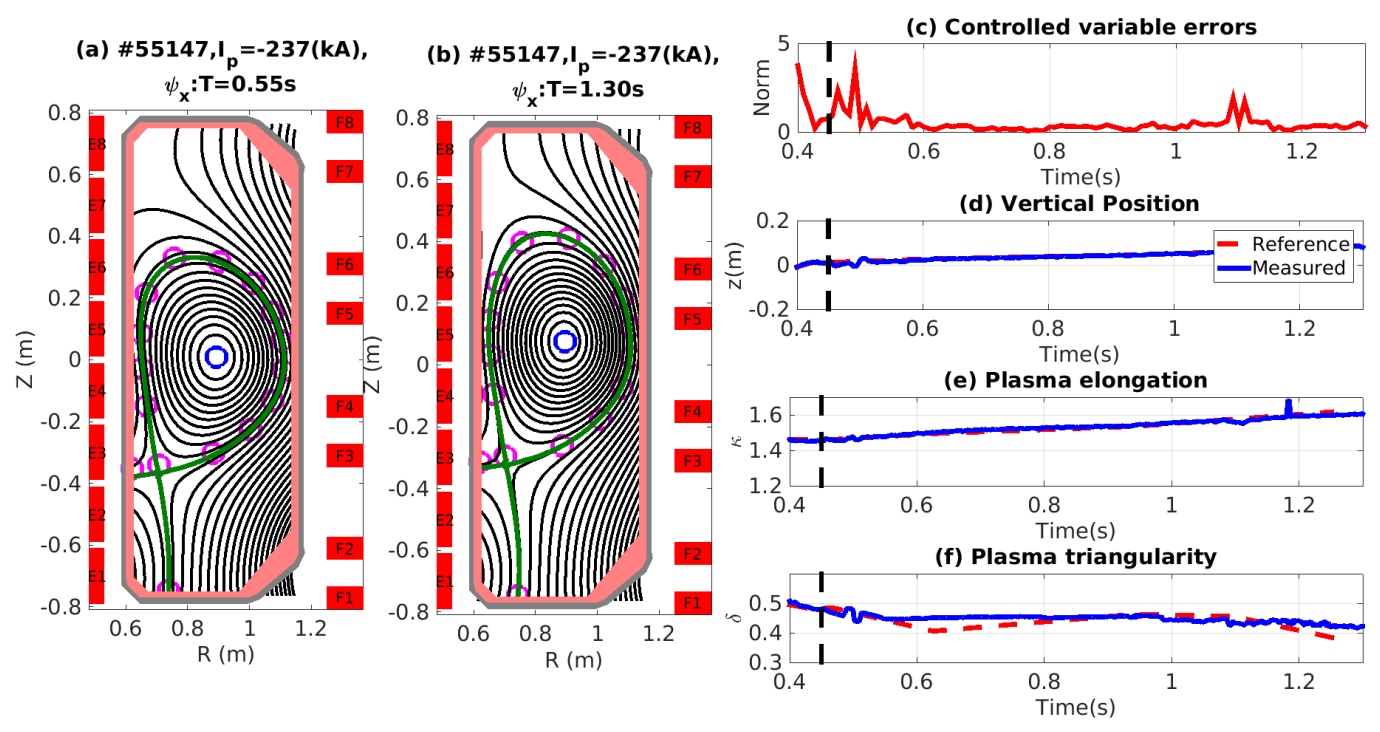

Figure 14: Performance of the generalised plasma position and shape controller for controlling time variant divertor plasma configuration with optimised actively controlled variables (8) and controller gains for position $\left(K_{s p}=0.55\right.$ and $\tau_{s i}=$ $0.1 s)$ and shape control $\left(K_{s p}=0.3\right.$ and $\left.\tau_{s i}=0.01 s\right)$. (a) Poloidal flux contour $\psi_{x}$ (black lines) and (b) last closed flux surface (green line) at different time instants of the controller operation, obtained from RTLIUQE. (c) Time evolution of norm of the errors of the actively controlled variables. Reference and measurement of the plasma vertical position (d), elongation (e) and triangularity (f) obtained from FBTE and LIUQE. The pre-programmed plasma boundary is given by control points in magenta circles.

\section{Summary and conclusion}

A generalised plasma position and shape control algorithm, developed particularly for advanced plasma configurations, has been presented in this paper. A linearised plasma model (RZIp), re-derived here in its entirety, is used to study the coupling between the TCV hybrid PID controller and the new generalised plasma position and shape controller. The model is also used to verify the performance of the control algorithm as well as to optimise the control parameters for the controlled variables. A successful experimental implementation of the control algorithm has been demonstrated for both fixed and time varying plasma position and shape for limiter and divertor plasma discharges. The control design exhibits an improved performance with respect to the control of the plasma position and shape relative to the legacy TCV hybrid controller.

The generalised plasma position and shape controller has been successfully 

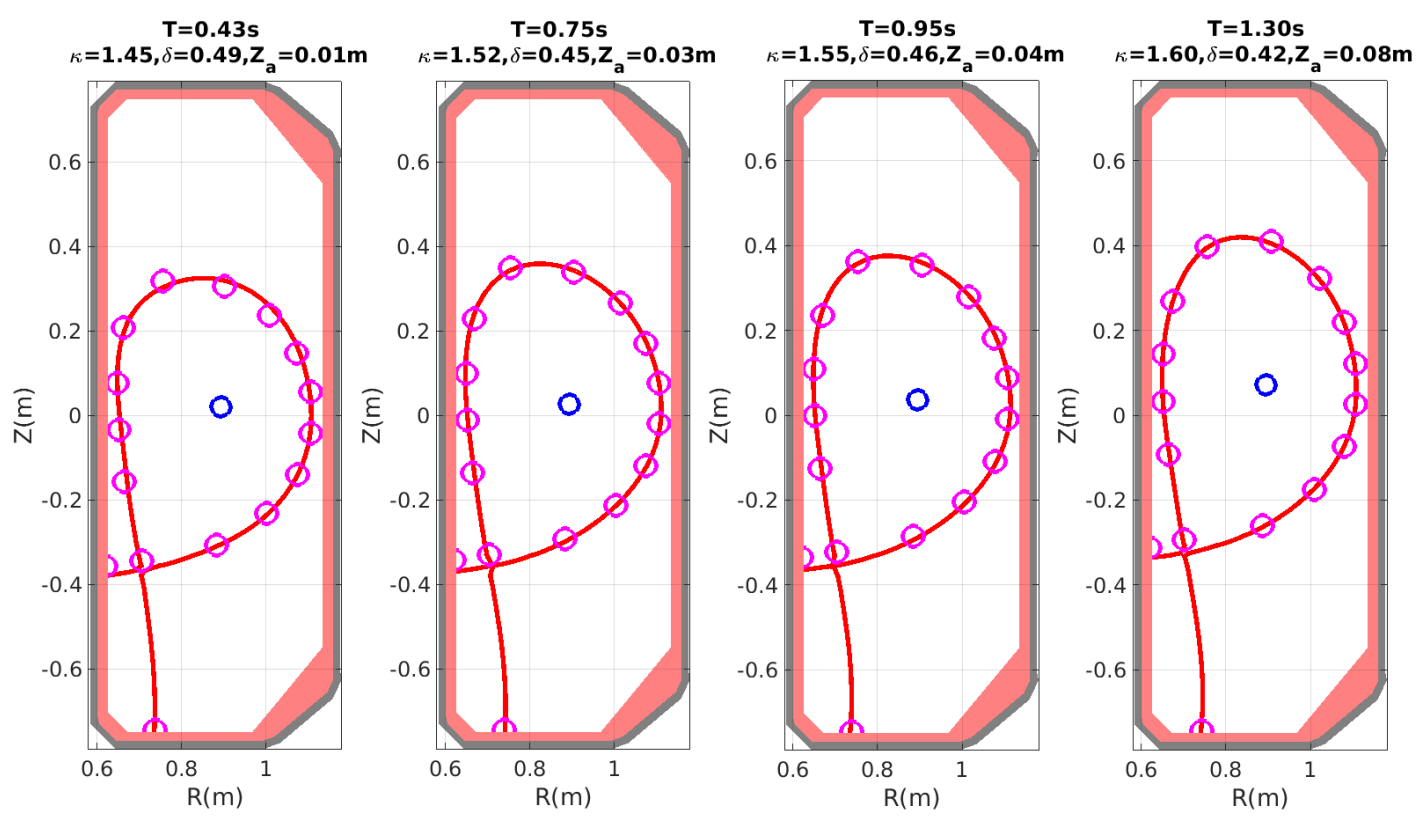

Figure 15: Separatrix (red line) and vertical position $Z_{a}$ of the plasma magnetic axis (blue circle) obtained from RTLIUQE for the plasma discharge 55147, at various time instances. $\kappa$ and $\delta$ represent the plasma elongation and triangularity obtained from LIUQE. The pre-programmed plasma boundary is denoted by magenta circles.

tested on various limiter and divertor plasma discharges involving complex changes in the position position as well as and shape during the discharge. A suitable range of controller gains has been experimentally found which provides good control of the plasma position without exciting instabilities, particularly the vertical instability. The controller can be optimised in an intuitive fashion, by promoting particular control variables through preferential weighting, while the singular-value decomposition formalism provides natural criteria for optimising the number of controlled parameters. Current limits in the poloidal field coils are respected by anti-windup techniques. A smooth behavior in time is ensured by the bumpless formalism.

In spite of considerable success in developing exotic plasma shapes without active control, future, more advanced developments in TCV - extending beyond the current empirical limits - are likely to depend on reliable shape control. The work described in this paper aims to be the cornerstone of these developments.

\section{Acknowledgement}

This work has been carried out within the framework of the EUROfusion Consortium and has received funding from the Euratom research and training programme 2014-2018 under grant agreement No 633053. The views and opinions expressed herein do not necessarily reflect those of the European Commission. 


\section{References}

[1] Ariola M et al. 2008 Magnetic Control of Tokamak Plasmas ISBN 978-184800-323-1

[2] Hofmann F et al. 1998 Physical Review Letters 81 2918-2921 ISSN 00319007 URL http://link.aps.org/doi/10.1103/PhysRevLett.81.2918

[3] Reimerdes H et al. 2000 Plasma Physics and Controlled Fusion 42629 ISSN 0741-3335 URL http://iopscience.iop.org/0741-3335/42/ 6/302\{\%\}5Cnhttp://iopscience.iop.org/0741-3335/42/6/302/pdf/ 0741-3335\{_\}42\{_\}6\{_\}302.pdf $\{\%$ GCnhttp://iopscience.iop.org/ $0741-3335 / 42 / 6 / 302$ ?rel $=$ ref $\{\&\}$ relno $=2$

[4] Martynov A N et al. 2005 Plasma Physics and Controlled Fusion 47 17431762 ISSN 0741-3335 URL http://stacks .iop.org/0741-3335/47/i=10/ $a=009$ ?key=crossref .ec75ac820a25668fa3c13d0c72398d52

[5] Pochelon A et al. 1999 Nuclear Fusion 39 1807-1818 ISSN 00295515 URL http://stacks.iop.org/0029-5515/39/i=11Y/a=321?key= crossref.5e7d6e5badb2bd58a36c0b6226eb444b

[6] Piras F et al. 2009 Plasma Physics and Controlled Fusion 51055009 ISSN 0741-3335 URL http://stacks.iop.org/0741-3335/51/i=5/a=055009? key=crossref .a281e046d21242eba91cedfc60587859

[7] Theiler C et al. 2017 Nuclear Fusion Submitted 1-15

[8] Reimerdes H et al. 2016 TCV Experiments towards the Development of a Plasma Exhaust Solution 26th IAEA Fusion Energy Conference - IAEA CN234 Contribution pp 1-8 URL https://conferences.iaea.org/indico/ event/98/session/36/contribution/908.pdf

[9] Paley J I et al. 2007 Plasma Physics and Controlled Fusion 49 17351746 ISSN 0741-3335 URL http://stacks .iop.org/0741-3335/49/i=10/ $\mathrm{a}=010$

[10] Ariola M et al. 2002 IEEE Transactions on Control Systems Technology 10 646-653 ISSN 10636536

[11] Ariola M et al. 2005 IEEE Control Systems Magazine 25 65-75 ISSN 02721708 URL http://ieeexplore.ieee.org/document/1512796/

[12] De Tommasi G et al. 2013 Shape control with the XSC during plasma current ramp-up and ramp-down at the JET tokamak Proceedings of the IEEE Conference on Decision and Control pp 366-371 ISBN 9781467357173 ISSN 01912216 
[13] Hutchinson I H et al. 1996 Fusion Science and Technology 30 137-150

[14] Ambrosino G et al. 2008 IEEE Transactions on Control Systems Technology 16 1101-1111 ISSN 10636536

[15] Ambrosino G et al. 2008 IEEE Transactions on Plasma Science 36 834-840 ISSN 00933813

[16] Hofmann F et al. 1988 Nucl. Fusion 28 ISSN 0029-5515 URL http: //iopscience.iop.org/0029-5515/28/10/014

[17] Moret J M et al. 2015 Fusion Engineering and Design 91 1-15 ISSN 09203796 URL //www.sciencedirect.com/science/article/pii/ S0920379614005973

[18] Ferron J R et al. 1998 Nuclear Fusion 38 1055-1066 ISSN 0029-5515 URL http://stacks.iop.org/0029-5515/38/i=7/a=308

[19] Coda $\mathrm{S}$ et al. 2013 Nuclear Fusion $\mathbf{5 3} 104011$ ISSN 00295515 URL http://iopscience.iop.org/0029-5515/53/10/ 104011\{\%\}5nhttp://iopscience.iop.org/0029-5515/53/10/104011/ $\{\%\} 5$ Cnhttp://iopscience.iop.org/0029-5515/53/10/104011/pdf/ $0029-5515\left\{_{-}\right\} 53\left\{\_\right\} 10\left\{_{-}\right\} 104011 . p d f$

[20] Favre A et al. 1997 Control of highly vertically unstable Plasmas in TCV with internal Coils and Fast Power Supply 19th Symposium on Fusion Technology pp 7-10 URL https://infoscience.epfl.ch/record/123685

[21] Hofmann F 1988 Computer Physics Communications 48 207-221 ISSN 00104655 URL //www.sciencedirect.com/science/article/pii/ 0010465588900410

[22] Hofmann F et al. 1995 Plasma Shape Control in TCV using MGAMS 22rd EPS Conference vol. 19C (Bournemouth) pp II-101

[23] Paley J I et al. 2010 Architecture and commissioning of the TCV distributed feedback control system Conference Record - 2010 17th IEEE-NPSS Real Time Conference, RT10 ISBN 9781424471096

[24] Felici F et al. 2014 Fusion Engineering and Design 89 166-176 ISSN 09203796

[25] Le H B et al. 2014 Fusion Engineering and Design 89 155-164 ISSN 09203796 URL http://www.sciencedirect.com/science/article/pii/ S0920379613006996

[26] De Tommasi G et al. 2011 Fusion Science and Technology 59 486-498 ISSN 15361055 
[27] Ambrosino R et al. 2015 Design and nonlinear validation of the ITER magnetic control system 2015 IEEE Conference on Control Applications (CCA) pp 1290-1295 ISBN 9781479977871 URL http://ieeexplore.ieee.org/ document/7320790/

[28] Albanese R et al. 2016 A MIMO architecture for integrated control of plasma shape and flux expansion for the EAST tokamak 2016 IEEE Conference on Control Applications (CCA) pp 611-616 ISBN 9781509007554

[29] Skogestad S et al. 2005 Multivariable feedback control: analysis and design ISBN 9780470011676

[30] Anand H et al. 2017 Nuclear Fusion 57056005 URL http://stacks .iop. org/0029-5515/57/i=5/a=056005

[31] Hofmann F et al. 1990 Nuclear Fusion 302013 ISSN 0029-5515 URL http: //stacks.iop.org/0029-5515/30/i=10/a=003

[32] Coutlis A et al. 1999 Nuclear Fusion 39 663-683 ISSN 0029-5515 URL http://stacks.iop.org/0029-5515/39/i=5/a=307

[33] Albanese R et al. 2015 CREATE-NL+: A robust control-oriented free boundary dynamic plasma equilibrium solver Fusion Engineering and Design vol 96-97 pp 664-667 ISSN 09203796

[34] Aström J et al. 1997 Computer Controlled Systems vol 53 ISBN 9788578110796 (Preprint arXiv:1011.1669v3)

\section{Appendix}

\subsection{Time varying controller design}

The output of the controller, $U_{k}$, at the switching sample $k_{s}$ is defined as:

$$
U_{k}=u_{k}^{i+1}+\left(u_{k_{s}-1}^{i}-u_{k_{s}-1}^{i+1}\right) F\left(k-k_{s}\right)
$$

where the indexes $i$ and $i+1$ denote the latter and former controllers, $F\left(k-k_{s}\right)=$ $e^{-\left(k-k_{s}\right) \tau}$ is a decaying exponential function, $\tau$ determines the time constant of bumpless transfer.

\subsection{Anti windup and bumpless transfer}

In general, the state space representation of the controller is given as

$$
\begin{gathered}
\dot{x_{c}}=F x_{c}+G y \\
u=C x_{c}+D y
\end{gathered}
$$




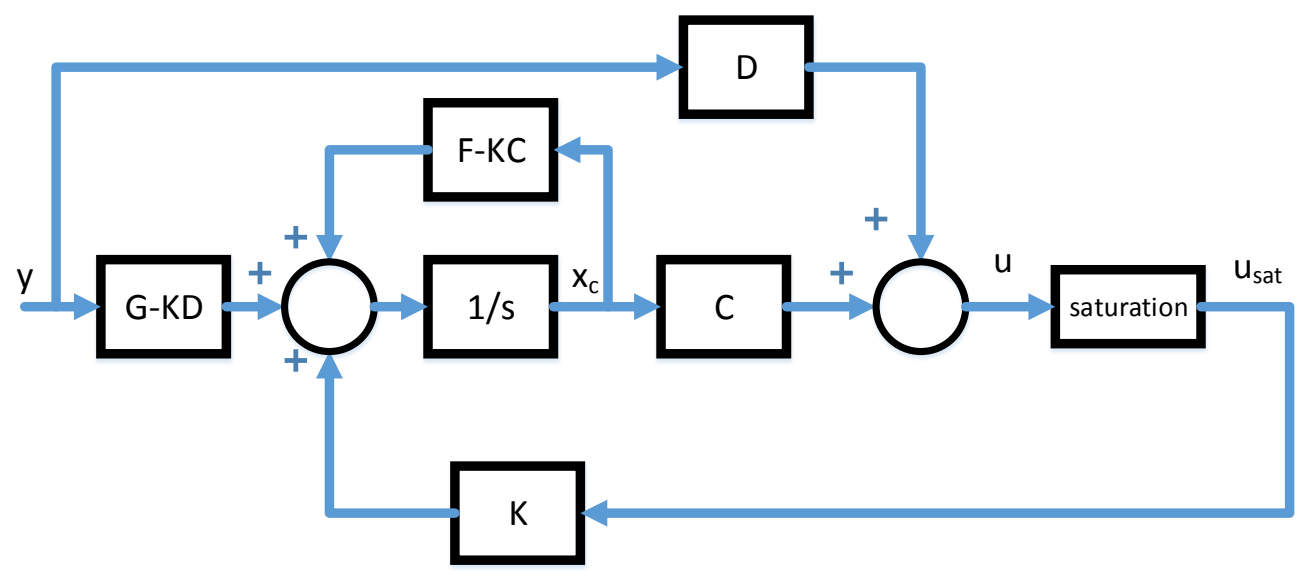

Figure 16: Block diagram of the controller showing the modification to avoid anti windup.

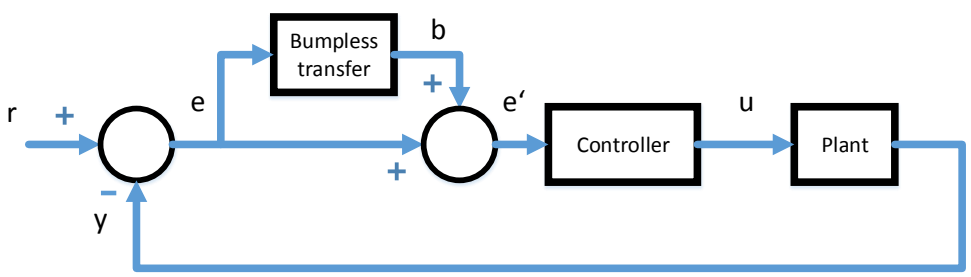

Figure 17: Block diagram of the controller showing the bumpless transfer implementation.

where $x_{c}$ represents the state and $F, G, C$ and $D$ are the state space matrices, $y$ and $u$ are the input and output of the controller. The state and the control signal can continue to evolve, although the influence on the process is restricted because of saturation.

A general state space controller can be formulated which avoids the windup problem; in this case, the control law is rewritten as indicated in figure 16 . This can be expressed as follows:

$$
\begin{aligned}
\dot{x} & =F x_{c}+G y+K\left(u_{s a t}-C x_{c}-D y\right) \\
& =(F-K C) x_{c}+(G-K D) y+K u \\
& =F_{0} x_{c}+G_{o} y+K u_{s a t}
\end{aligned}
$$

If the system of $(12)$ is observable, the matrix $K$ can always be chosen such that $F_{0}=F-K C$ has stable eigenvalues [34]. This ensures that the state assumes a proper value when the controller output saturates and thus prevents the problem of windup.

The discontinuity introduced by the switching between the position control 
of the TCV hybrid controller to the position control performed by the designed controller is avoided by developing a bumpless transfer mechanism shown in figure 17. Through the addition of a modification $b$ to the error on each controller variable $e$,

$$
e^{\prime}=e+b
$$

where $e^{\prime}$ is the modified error to the controller. The modification $b$, providing a smooth transition between subsequent controllers, is defined as follows:

$$
b= \begin{cases}-e & \forall t<t_{s} \\ e_{t_{s}}(L(s)-I) & \forall t \geq t_{s}\end{cases}
$$

where $e_{t_{s}}$ is the error between the controllers at the switching time $t_{s}$ and $L(s)$ is a first order filter in the Laplace domain $s$, with time constant $\tau$,

$$
L(s)=\frac{1}{\tau s+1}
$$

The formalism defined by 13 ensures that, before the activation, the controller artificially witnesses a zero error and tends smoothly to the active error according to the chosen time constant defined by 115 .

\section{$6.3 \quad$ RZIP}

The circuit model representation of the structures (active and passive conductors) takes the following form:

$$
\begin{gathered}
\vec{V}_{a}=M_{a a} \overrightarrow{\dot{I}}_{a}+M_{a v} \overrightarrow{\dot{I}}_{v}+R_{a} \vec{I}_{a}+\frac{d}{d t}\left(M_{a p} I_{p}\right) \\
0=M_{v v} \overrightarrow{\dot{I}}_{v}+M_{v a} \overrightarrow{\dot{I}}_{a}+R_{v} \vec{I}_{v}+\frac{d}{d t}\left(M_{v p} I_{p}\right)
\end{gathered}
$$

where $\mathrm{M}$ denotes a mutual inductance matrix, with indexes $\mathrm{a}, \mathrm{v}$, and $\mathrm{p}$ referring to "active conductor", "vessel" (passive conductor), and "plasma", respecively. $R_{a}$ and $R_{v}$ are the active and passive conductor resistance matrices, $\vec{I}_{a}$ and $\vec{I}_{v}$ are the vectors of currents in active and passive conductors, $I_{p}$ is the plasma current and $\vec{V}_{a}$ is the vector of voltages for active conductor.

The plasma current dynamic response is governed by a similar circuit equation, which treats the plasma as a single circuit consisting of a distributed array of conducting elements. The plasma response equation can be written in the form

$$
0=\frac{d}{d t}\left(M_{p a} \vec{I}_{a}\right)+\frac{d}{d t}\left(M_{p v} \vec{I}_{v}\right)+\frac{d}{d t}\left(L_{p} I_{p}\right)+R_{p} I_{p}
$$

where $L_{p}$ is the plasma self-inductance and $R_{p}$ the plasma resistance matrix.

Next we tackle the time derivatives of the radial and vertical force balance equations, neglecting the plasma inertia. The radial force balance consists of two 
components: the Lorentz force acting on the plasma due to the vertical magnetic field and the hoop force generated by the plasma pressure in toroidal geometry.

$$
\begin{gathered}
\frac{d}{d t}\left(m_{p} \frac{d^{2} R}{d t^{2}}\right)=\frac{d}{d t}\left(\frac{\mu_{o} I_{p}^{2}}{2} \Gamma+2 \pi R I_{p} B_{z}\right)=0 \\
\frac{d}{d t}\left(m_{p} \frac{d^{2} Z}{d t^{2}}\right)=\frac{d}{d t}\left(-2 \pi R I_{p} B_{r}\right)=0
\end{gathered}
$$

where $m_{p}$ is the inertia of the plasma, $R$ and $Z$ represent the radial and vertical positions of the current centroid, $B_{r}$ and $B_{z}$ are the radial and vertical magnetic fields produced by the conductors, $\Gamma$ is a function of the plasma self inductance, $l_{i}$, and plasma beta, $\beta_{p}$.

Linearisation of the set of equations around an equilibrium point, $\vec{I}_{a}=\vec{I}_{a o}(t)+$ $\delta \vec{I}_{a}, \vec{I}_{v}=\vec{I}_{v o}(t)+\delta \vec{I}_{v}, I_{p}=I_{p o}(t)+\delta I_{p}, \vec{V}_{a_{0}}(t)+\delta \vec{V}_{a}, R=R_{o}+\delta R, Z=Z_{o}+\delta Z$ and $\Gamma=\Gamma_{o}+\delta \Gamma$.

Definition for the elements of the $M$ and $R$ matrices,

$$
\begin{gathered}
M_{p R}=\frac{\partial L_{p}}{\partial R}+\frac{2 \pi R_{o} B_{z o}}{I_{p o}}, M_{R p}=\frac{2 \pi R_{o} B_{z o}}{I_{p o}}+\mu_{o} \Gamma_{o} \\
M_{R R}=\frac{\mu_{o}}{2} \frac{\partial \Gamma}{\partial R}+\frac{2 \pi R_{o}}{I_{p o}} \frac{\partial B_{z}}{\partial R}+\frac{2 \pi B_{z o}}{I_{p o}}, M_{Z Z}=-\frac{2 \pi R_{o}}{I_{p o}} \frac{\partial B_{r}}{\partial Z}, \\
M_{Z R}=-\frac{2 \pi R_{o}}{I_{p o}} \frac{\partial B_{r}}{\partial R}, M_{R Z}=\frac{2 \pi R_{o}}{I_{p o}} \frac{\partial B_{z}}{\partial Z} \\
R_{R p}=\frac{2 \pi R_{o}}{I_{p o}} \dot{B_{z o}}+\frac{\mu_{o} \Gamma_{o} I_{p o}}{I_{p o}}+\mu_{o} \dot{\Gamma_{o}}, \\
R_{R R}=\frac{\dot{\mu_{o}}}{2} \frac{\dot{I_{p o}}}{I_{p o}} \frac{\partial \Gamma}{\partial R}+\frac{2 \pi B_{z o}}{I_{p o}}, \\
R_{v R}=R_{R v}=\frac{\dot{I_{p o}}}{I_{p o}} \frac{\partial M_{v p}}{\partial R}, R_{a R}=R_{R a}=\frac{\dot{I_{p o}}}{I_{p o}} \frac{\partial M_{a p}}{\partial R}, \\
R_{v Z}=R_{Z v}=\frac{\dot{I_{p o}}}{I_{p o}} \frac{\partial M_{v p}}{\partial Z}, R_{a Z}=R_{Z a}=\frac{\dot{I_{p o}}}{I_{p o}} \frac{\partial M_{a p}}{\partial Z}, \\
R_{p R}=\frac{\partial R_{p}}{\partial R}+\frac{2 \pi R_{o} B_{z o}}{I_{p o}}-\frac{2 \pi R_{o} B_{z o}}{I_{p o}^{2}} \dot{I_{p o}}, \\
S_{R}=-\frac{\mu_{o}}{2} \frac{\partial \Gamma}{\partial l_{i}}\left(I_{p o} \dot{\delta} L_{i}\right)-\frac{\mu_{o}}{2} \frac{\partial \Gamma}{\partial \beta_{p}}\left(I_{p o} \dot{\delta} \beta_{p}\right)-\frac{\mu_{o}}{2} \frac{\partial \Gamma}{\partial l_{i}}\left(\dot{I_{p o}} \delta L_{i}\right)-\frac{\mu_{o}}{2} \frac{\partial \Gamma}{\partial \beta_{p}}\left(\dot{\left.I_{p o} \delta \beta_{p}\right)}\right.
\end{gathered}
$$

\subsection{Sign-bit Controller}

In order for the poloidal field coil to switch the current polarity, it is necessary to send to its power supply a digital signal, i.e., a sign bit of suitable polarity, magnitude and pulse width. Sign bits in TCV are pre-calculated in MGAMs [31] 
based on the feedfoward PF coil current requests and sent as feedfoward traces to the PF coil controllers. As the currents are based on FBTE calculations, which in turn are based on assumptions about the current profile - typically assumed to be Ohmic-like - substantial auxiliary heating can cause a significant departure from the predicted currents, in which case the pre-calculated sign bits may be inaccurate. If a current reaches zero and the relevant sign bit has not been issued, the coil current languishes at zero and the current evolution is different from the pre-programmed request. This problem is greatly exacerbated by the generalised plasma position and shape controller algorithm, which often requests coil currents that are very different from the pre-programmed ones. The signbit issue is an inherent hindrance to true real-time control, and its resolution was seen as an integral component of the shape controller development. The new, fully digital, distributed control system (SCD) presents an opportunity for the development of a sign bit controller based on the real time measurement of the poloidal field coil currents. An algorithm based on the following scheme has been developed in the Simulink block diagram environment and has been successfully tested experimentally. Two unique thresholds $\left(T h_{1}\right.$ and $T h_{2}, T h_{1}$ $\left.>T h_{2}\right)$ for the poloidal field coil currents are defined. Upon the crossing of the first threshold by the absolute value of a given poloidal field coil current, a sign-bit of opposite polarity is generated. When the poloidal field coil current crosses the second threshold (lower than the first one), a sign-bit of the same polarity as the previous sign-bit is generated. Further, when the poloidal field coil currents stays under the second threshold, alternating sign-bits separated by a predefined time interval are generated until the poloidal field coil current switches polarity. 